\title{
Particle Attrition Mechanisms, their Characterisation, and Application to Horizontal Lean Phase Pneumatic Conveying Systems: A Review
}

\author{
B.A. Kotzur, R.J. Berry, S. Zigan, P. García-Triñanes \& M.S.A. Bradley
}

\section{Abstract}

Understanding particle attrition is vital to the optimisation of a wide range of industrial processes. Lean phase pneumatic conveying is one such process, whereby the high energy particle impacts can cause undesirable loss in product quality or change in bulk behaviour. The attrition process is resolved into a material function and a process function; the combination of these functions dictate the attrition mechanism present, and the magnitude of failure observed. Subsequently, the forces applied to the particles are examined within the context of lean phase pneumatic conveying. Finally, empirical and numerical models are reviewed along with comments on experimental method.

To summarise some of the findings of this review: the requirement of standardised test equipment is recognised in order to compare the wide variety of particulate materials under comparable loading conditions; stronger correlation between the results obtained from different particle attrition test methods is required; and finally, seldom are the manufacturing conditions (where applicable) linked to the particulate attrition behaviour.

\section{Keywords}

Particle Attrition, Pneumatic Conveying, Lean Phase 


\section{Contents}

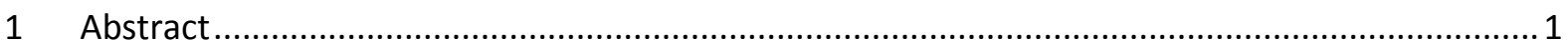

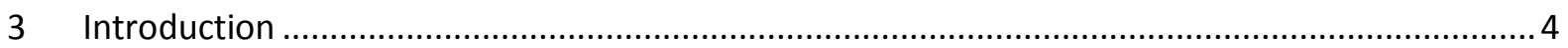

4 Particle Attrition within Lean-Phase Pneumatic Conveying ...................................................... 6

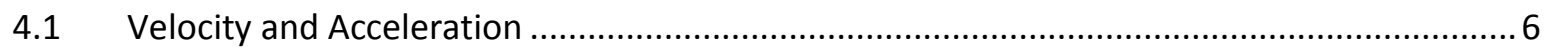

4.2 Pressure Drop...................................................................... Error! Bookmark not defined.

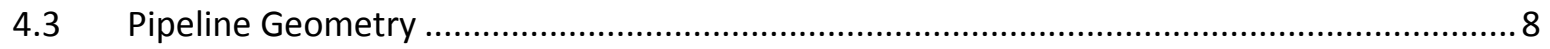

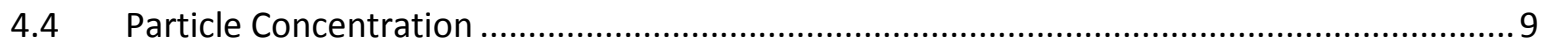

4.4.1 System ....................................................................... Error! Bookmark not defined.

4.4.2 Mesoscale ...................................................................... Error! Bookmark not defined.

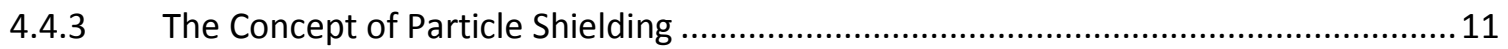

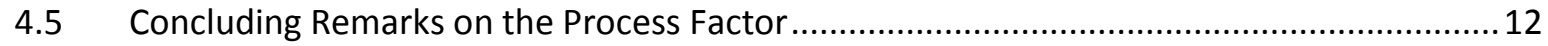

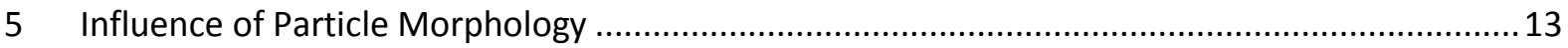

$5.1 \quad$ Failure Modes ...................................................................... Error! Bookmark not defined.

5.1.1 Particle Plasticity ............................................................. Error! Bookmark not defined.

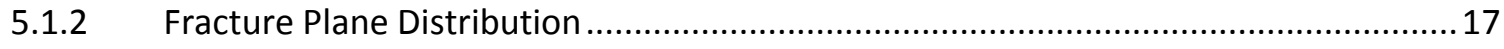

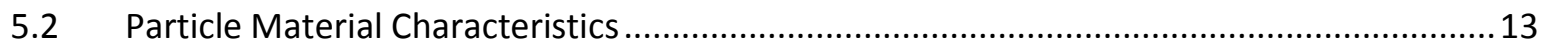

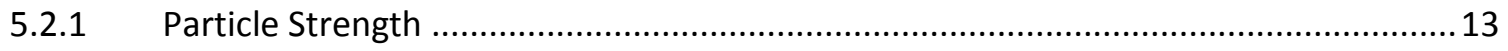

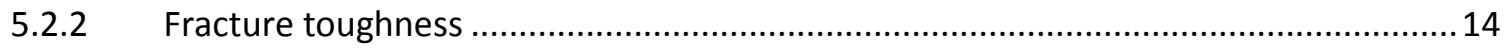

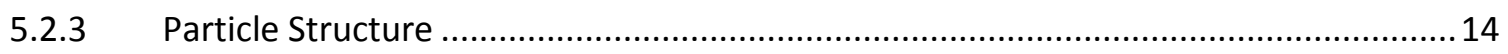

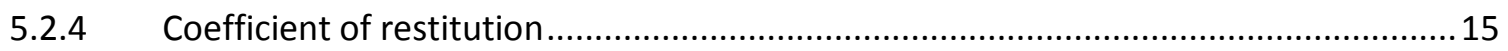

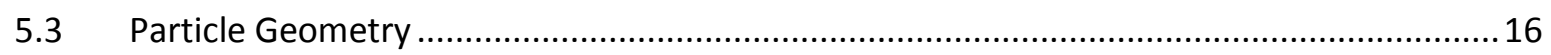

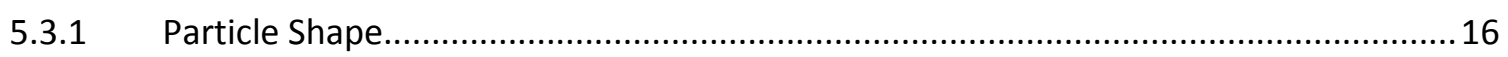

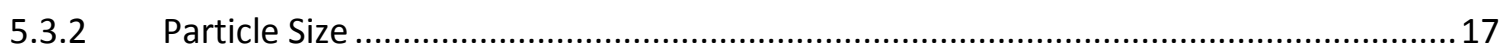

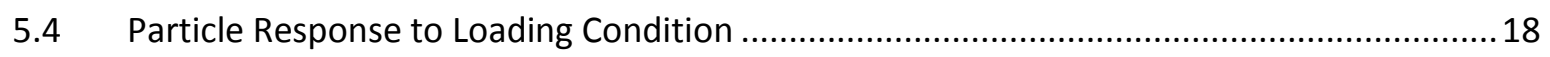

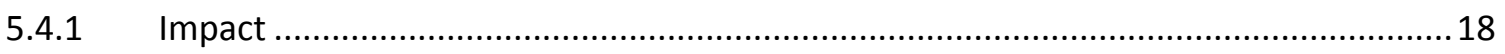

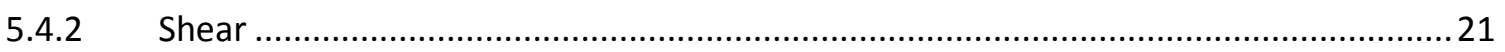

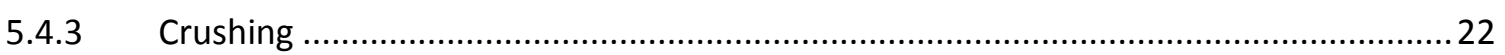

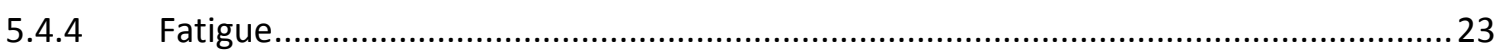

5.5 Experimental Techniques for Measuring Particle Attrition ................................................24

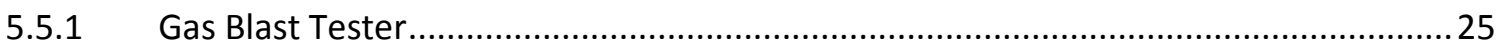

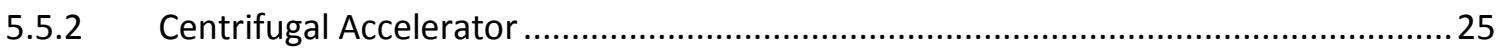

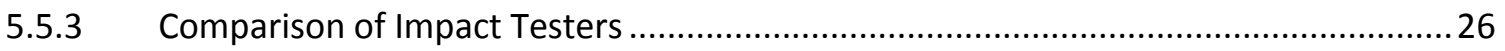

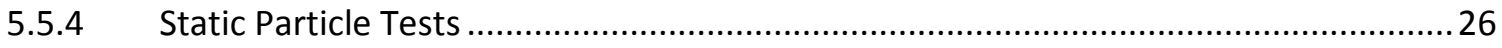

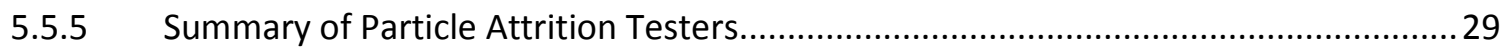

5.6 Concluding Remarks on the Material Factor .................................................................. 32 
6 Modelling of Particle Attrition in Lean Phase Pneumatic Conveying

.33

6.1 Derivation from First Principles

Error! Bookmark not defined.

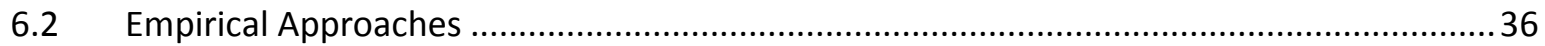

6.3 Concluding Remarks on Modelling Approaches ................................................................ 40

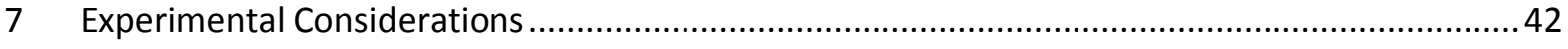

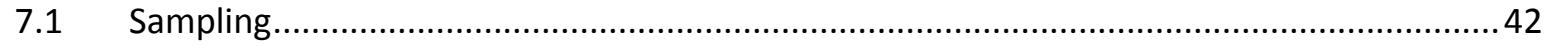

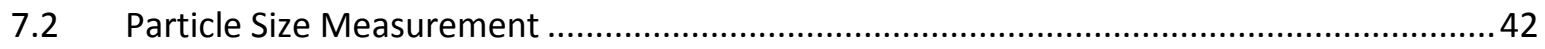

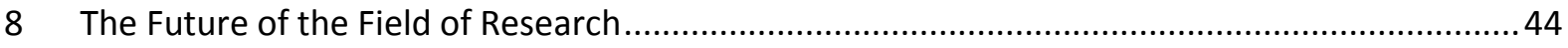

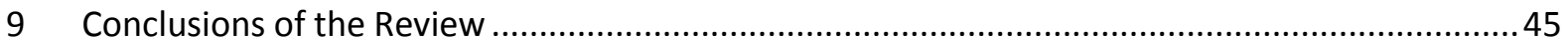




\section{Introduction}

Breakage of particles within industrial processes can be either desirable (comminution) or undesirable (attrition). A wide range of processes involve handling steps that have the potential to break the particles concerned. If breakage is excessive, it can lead to a number of undesirable repercussions including (but not limited to):

- Loss in perceived quality

- Customer perception (tea leaves, sugar crystals)

- Increase in dust generation

- Increased possibility of dust explosions (and secondary dust explosions if illmanaged)

- Increased expense in dust management and handling systems

- Loss in functionality and product performance

- Processes sensitive to particle surface area (catalytic particles)

- Change in flow behaviour (increased fines leading to difficulties in emptying hoppers)

- Changes in mixing behaviour (increased propensity for segregation)

$\circ$ Changes in bulk density (error in volumetric dosing processes)

This review will explore the state-of-the-art on research regarding particle attrition, and subsequently look at the applications and studies surrounding lean phase pneumatic conveying. The subject is divided first into the process influences and the material influences affecting particle attrition, followed by existing models published to predict these interactions. Finally, experimental considerations are presented followed by exploration of where progress is required in the field. Key review works are summarised in the proceeding paragraphs to lay the foundation on which this work will build.

Bemrose and Bridgwater [1] covered an extensive range of topics, broadly categorised under four themes: the nature and occurrence of attrition, powder attrition mechanisms, characterisation and assessment of particulate attrition, and attrition testing methods. Two key challenges are highlighted at the conclusion of the review:

1. Specification of a useful, useable and representative test method, in which the attrition mechanism is well understood, within a test environment that is simple and well-defined.

2. Specification of a reproducible and satisfactory method of assessing attrition in terms of its extent and nature.

Both of these challenges are defined within a test method that is ideally accelerated in order to maximise utility. Shear testing and impact testing are concluded to have clear importance, however the attrition mechanisms present in such tests do not share a strong correlation with the mechanisms present in lean phase pneumatic conveying. Furthermore, the number of loading scenarios contained within industrial processes are diverse, adding another dimension to the complexity in predicting attrition behaviour.

Hutchings [2] recognised that surface erosion and particulate attrition are both results of the same contact event. Abrasive and erosive wear of ductile and brittle surfaces were addressed. The mechanisms responsible for material removal vary significantly between the two surface material types. Material properties of fundamental importance were determined to be the fracture toughness, $\mathrm{K}_{\mathrm{c}}$, and the hardness, $\mathrm{H}$, of the surface material, and the hardness of the particles. In applying this to the concept of particle attrition, the results of a particle-wall contact may be viewed 
in terms of damage to either the particle or wall, or both, depending on the properties of each material and the method by which a contact takes place. Hutching also notes the likelihood of a fundamental relationship between surface wear and particle attrition (or particle wear) in such scenarios.

Breakage of agglomerates within the granulation process is reviewed by Reynolds et al. [3]. Breakage is addressed at the process scale (bulk behaviour), and at the granule scale (particle behaviour), and how the variables in the granulation process affect these. The manufacturing method of granules have significant influences on the breakage behaviour it will display. Factors to be considered include: properties of the binder (viscosity, surface tension, amount of binder added, and contact angle with primary particles), size and shape of the primary particles, and granulation process variables (such as agitation intensity, granulation time, and binder addition method). Furthermore, different agglomerate types are considered; noted to contain different types of bonds between the primary particles. Whilst this review does not address the attrition of agglomerates within pneumatic conveying systems, it does provide valuable insight into the complex particle structure of agglomerates, and subsequently, their specific failure mechanisms.

Now, building on these previous works, this paper will summarise the current state of knowledge of the field to provide the reader with a concise understanding of particle attrition and its manifestation in lean phase pneumatic conveying systems. 


\section{Particle Attrition within Lean-Phase Pneumatic Conveying}

The studies reviewed in this section will account for the loading conditions imposed on particles within lean phase pneumatic conveying systems.

\subsection{Velocity and Acceleration}

In the context of particle attrition by impact, it has been repeatedly demonstrated that impact velocity is by far the most influential factor. It is therefore essential to understand the operating parameters which affect the particle velocity within the pipeline.

The superficial air velocity is obtained by neglecting the presence of the solid particles within the pipeline, and is often used as an operating condition to define the system. To achieve a lean phase pneumatic conveying condition, superficial air velocities are typically in the range of 10 to $45 \mathrm{~m} / \mathrm{s}$ [48]. Particle velocity during pneumatic conveying is the velocity at which a particle travels longitudinally along the pipeline using a global coordinate system as a frame of reference. It is widely accepted that in order to minimise the degree of particle attrition occurring within a pipeline, a superficial air velocity as close as is possible to the saltation velocity should be used.

The difference between the superficial air velocity and the particle velocity is known as the slip velocity. A number of works found that the slip velocity is negligible under certain conditions, and therefore assume the superficial air velocity is equal to the particle velocity (for example, see [9]). A number of different methods have been adopted to measure particle velocity (enabling the calculation of slip velocity), including:

1. High speed photography $[10,11]$

2. Two photodiodes separated by a known distance $[12,13]$

3. Photodiode coupled with a vibration transducer separated by a known distance [14]

4. Electrostatic sensing approaches $[15,16]$

5. Laser Doppler Anemometry $[8,17]$

6. Optical Fibre Probe [18]

7. Radiometric Sensors [19]

When considering a pipeline geometry where straight sections of pipe are insufficiently long to accommodate steady-state flow, the acceleration profile of the particulate material is required. Mendies et al. [20] looked at the effect of suspension density on the acceleration of particles within a pipeline. Through studying the dilute flow of plastic particles with pin electrodes, the mean axial particle velocity was determined. The particle velocities were plotted against their corresponding air velocities, clearly showing the presence of a slip velocity (Figure 1). It was demonstrated that the solids loading ratio had negligible effect on the fully expanded flow slip velocity of the particles (Figure 2). With respect to the length of the acceleration zone, all materials tested required the full horizontal pipe length of $11 \mathrm{~m}$ to reach a steady-state flow. 


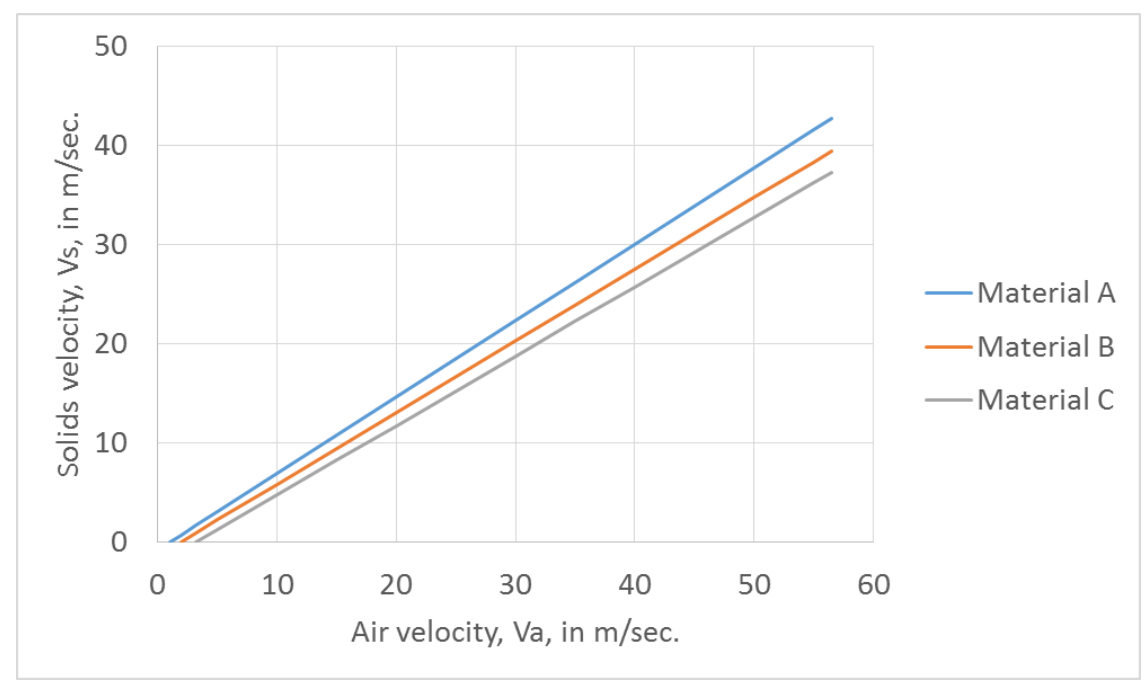

Figure 1: Particle Velocity plotted against Air Velocity for three Plastic Materials adapted from Mendies et al. [20].

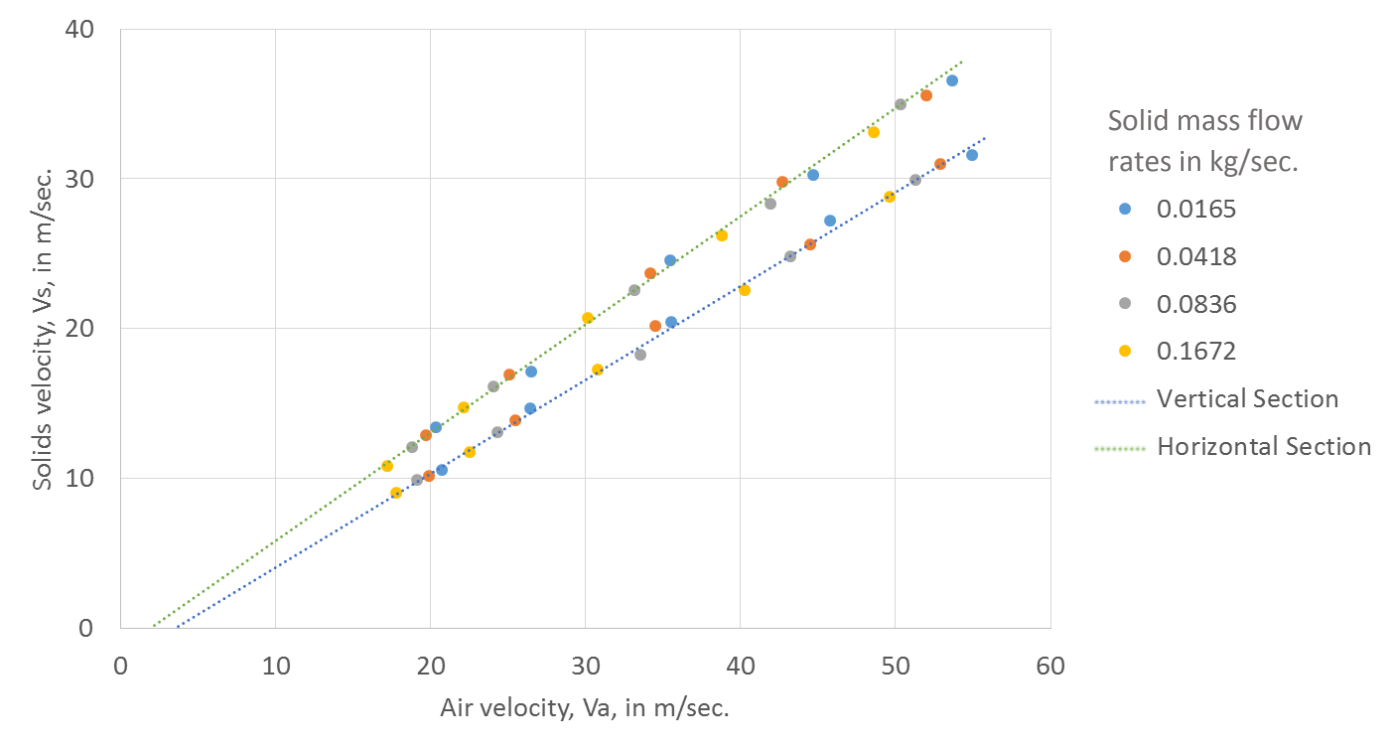

Figure 2: Particle Velocity vs Air Velocity across a range of Solids Loading Ratios for Vertical and Horizontal Sections adapted from Mendies et al.[20]

A model to describe the acceleration of particles after a bend in lean phase pneumatic conveying systems was presented by Bradley et al. [21]. The calculation takes into account a moving bed of material (strand) which progressively loses mass to a clear bore flow of air (Figure 3). The interaction between these two phases is governed by the shear force imposed across the face of the moving bed exposed to the clear bore. The force balance used in the calculation is also shown in Figure 3. This model demonstrated a reasonable correlation with experimental results (superficial air velocity range and suspension density range; $21-30 \mathrm{~m} / \mathrm{s}$, and $8-30 \mathrm{~kg} / \mathrm{m}^{3}$ respectively), with a mean absolute error of $8 \%$. Particle size and density were deemed to have minimal effect on the acceleration length. 


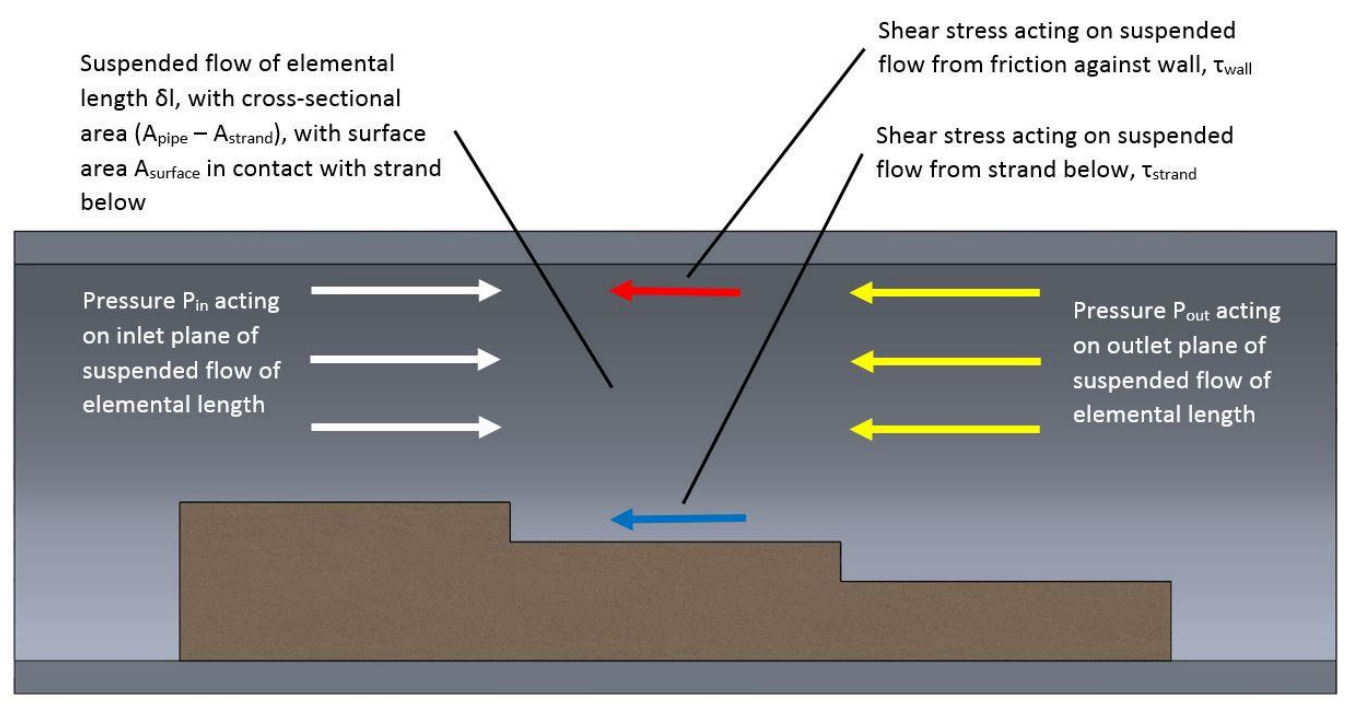

Figure 3: Strand Flow Force Balance adapted from the Acceleration Model by Bradley et al.[21]

\subsection{Pipeline Geometry}

Pipeline geometry includes the internal diameter, material, internal finish quality, alignment, length of the straight sections of pneumatic conveying pipeline, and the geometry of the bends and couplings (these topics shall also be addressed with respect to particle concentration in Section 4.3, and consolidated in Section 4.4). The feeding arrangement or receiving arrangement shall not be addressed.

The pipe diameter used in the majority of studies is small, ranging from 0.75 to 2 inches $[4,6,22]$, however some account for pipe bores as large as 4 inches in diameter [23]. One possibility for this trend is that an exponentially greater volumetric air flow rate is required to achieve the same conveying conditions with a larger bore size. Whilst use of small pipe diameters is more economically viable, a scale-up procedure is required to calculate the flow conditions expected in pipe systems of a larger bore size. Examples of scale-up models may be found within the works of Wypych and Arnold [24], Wypych [25], and Bradley [23].

The length of straight sections of pipe is expected to inflict minimal damage on the conveyed particles in comparison to that occurring at the pipe bends [4], and therefore is acknowledged to cause little impact on attrition.

It has been demonstrated by Lagrangian simulation, that the wall roughness does alter the particle rebound behaviour following particle-wall contacts [26] for $50 \mu \mathrm{m}$ and $100 \mu \mathrm{m}$ spherical glass particles. Increasing wall roughness was found to reduce mean particle velocity (increase slip velocity), and reduction of 'particle-free zone' within the pipe cross section. Furthermore, these results were influenced by particle size. These results are supported by the experimental work of Sommerfeld and Kussin [27], albeit with a rectangular channel. The channel was $35 \mathrm{~mm}$ high and 350 $\mathrm{mm}$ wide with a length of $6 \mathrm{~m}$. Surface roughness was tested across a 2-17 $\mu \mathrm{m}$ range, and mean particle sizes of $60-625 \mu \mathrm{m}$, at a conveying velocity of approximately $20 \mathrm{~m} / \mathrm{s}$. They concluded that when the surface roughness of the channel wall was increased, the particle distribution across the channel cross-section became more uniform. The same trend was observed with increasing particle size. The effect of wall roughness decreases with decreasing particle size. Finally, the measured slip 
velocity increased with increasing wall roughness. While there are no studies that link directly the effect of wall roughness on the attrition observed in lean phase pneumatic conveying systems, the aforementioned studies do yield valuable insight into the particle-wall contact conditions required for prediction of attrition.

The bends have been widely accepted to be the location of the majority of attrition within lean phase pneumatic conveying systems as previously stated. A number of studies have been conducted into determining the influence of bend geometry on particle attrition behaviour $[5,22,28,29]$. The variable of interest in these studies has been the bend $r / D$ ratio (radius of the centreline of the bend divided by the diameter of the pipe). Many studies also refer to short radius ( $r / D \approx 3$ to 5 ) and long radius ( $r / D \approx 9$ to 12 ). Conflicting reports have been produced, with some results indicating that long radius bends produce more particle attrition [5], and others indicating that short radius bend produce more attrition $[22,28,29]$. The argument made in favour of increased degradation in long radius bends is that particles are expected to be subjected to a higher number of impacts. Conversely, higher impact angles are expected in short radius bends, theoretically resulting in increased particulate attrition. Particle shape can be a significant factor when considering particlewall interaction, as demonstrated by Deng et al. [30]. These observations may contribute to an underlying relationship with the impact angle expected within different lean phase pneumatic conveying bend types.

\subsection{Particle Concentration}

Particle concentration measurement techniques shall first be considered, followed by acknowledgement of variation of the particle concentration across the conveying pipe cross-section. Finally, the concept of particle shielding shall be addressed.

\subsubsection{Measurement Techniques}

Several techniques have been developed to measure the particle concentration within pneumatic conveying pipelines. These are largely addressed in the review works of Zheng and Liu [31], and Yan [32], and therefore a selection of techniques shall be summarised here.

\section{Averaged Measurement Methods}

If the air mass flow rate and the solids mass flow rate are monitored, the particle concentration throughout the pipeline can be defined as a dimensionless ratio of mass flow rates (also known as the solids loading ratio). This approach has the advantage of remaining constant along the length of the pipeline and has been widely used $[5,7,27,33,34]$. Measurement of this variable is facilitated with a loss-in-mass measurement of the feed hopper and air supply characteristics, and is typically between 0-15 for lean phase pneumatic conveying systems [35]. However, this criterion cannot be used to describe the conditions in a pipe bend in isolation, as the volume of fluid phase changes along the length of the pipe, and the residence time of the solid phase is specific to the pipeline geometry and conveying conditions.

Additionally, the volumetric loading ratio (dimensionless ratio of volumetric flow rates of solids to gas phase) can be calculated along the pipeline, local to online pressure tappings $[15,36]$. The value of this variable changes along the length of the pipeline to reflect the expansion of conveying air.

A hybrid of the previous two methods can also be adopted, whereby the ratio of the mass flow rate of solids to volumetric flow rate of the gas phase is used to characterise the particle concentration in the conveying line [37-39]. 


\section{Electrical Capacitance Tomography (ECT)}

Electrical Capacitance Tomography functions on the basis that as the amount of material in a pipeline increases, the measured capacitance across the pipe cross-sectional area will also increase. Sensing electrodes can take a number of forms including pins, rings and plates, mounted internally or externally (on insulated pipe) on the pipeline [32]. Whilst the resources required to implement this technique are relatively cheap, it is necessary to evaluate the influence of baseline drift, temperature of the conveyed phases, and moisture content of the conveyed phases, as in the work of Hu et al. [40]. Moreover, it is essential that the operator is aware of build-up of the solids phase in the sensing volume of the pipeline as this too will affect the readings obtained. Sensor structure is a key consideration in how two-phase flows are characterised. This is exemplified in the work of Yang and $\mathrm{Xu}$ [41], who compared two twin-array capacitance sensors: one with 4 electrodes and the other with 6 . It was concluded that while the 4-electrode sensor offers a higher data acquisition speed (making it suitable for high-velocity flows), the 6-electrode sensor can detect more complex flow patterns.

\section{Radiometric Sensors}

This method of particle concentration measurement can use sources such as low-energy gamma rays (such as Am-241) [32], or x-ray point sources [42] to inspect the entire pipe cross section. While there is only a weak effect of moisture content of the conveyed medium on the signal obtained [43], a key consideration of this approach is to design such a system that is capable of inspecting the entire pipe cross-section with the single-line attenuation nature of the source and sensor.

Alternative configurations include a scanning densitometer (unsuitable for transient measurements), multi-path configuration, and use of a single broad-beam source [32]. The use of a soft x-ray field source has proven compatible with dilute phase pneumatic conveying due to its much higher radiation attenuation [44].

\section{High-Speed Imaging / Particle Image Velocimetry (PIV)}

High speed imaging techniques for measuring particle concentration requires collection of a series of images or frames, which are used to calculate particle position and velocity across known time intervals. This method is best used to observe low-concentration particle flows [10] and detectable particle size is determined by the camera resolution. There is also the drawback of particle masking, where a particle travels in front of another from the perspective of the camera, hiding the latter.

Another approach used to determine the particle concentration is through using a single image captured over a longer exposure time, and thus obtain an image containing particle traces. These traces represent the particle diameter and velocity through the width and length of the trace respectively [45]. A more comprehensive review of PIV techniques can be found in the work of Miyazaki et al. [46].

\subsubsection{Particle Concentration across the Conveying Pipe Cross Section}

In addition to findings mentioned in Section 4.2, investigations of the cross-sectional particle concentration have found that in steady-state lean-phase conveying, it is greater in the lower half of the pipe [47]; such behaviour is also true of rectangular channels [48]. This has been largely attributed to the influence of gravity on the particle flow, leading to particles favouring a position closer to the bottom of the pipe. Additionally, the immediate straight pipe section following the bend exhibits a cross-sectional solids concentration described as the previously mentioned strandtype flow [21]. This is due to the material being forced to the outer wall of the pipeline in the bend section. 
Fokeer et al. [49] summarises the effects of pipe wall roughness, mass loading, and pipe bends on the cross-sectional concentration of solids in lean phase pneumatic conveying. It was reported that as pipe wall roughness increases, the gravitational settling of the solid particles is reduced while increasing the pipeline pressure drop. Increasing mass loading conditions resulted in a reduction of particle velocity fluctuations. Finally, bend orientation and geometry affect the formation of a particle strand in the post bend pipe section. If the conveyed solids have a sufficiently wide particle size distribution, particle size segregation will occur longitudinally in the post bend pipe section; this effect is more prominent at lower conveying velocities and higher mass loading ratios.

Particle pathing has also been tracked through a pipeline including a bend and other industrial equipment, using Positron Emission Particle Tracking (PEPT) [50-52]. This method involves labelling a particle with ${ }^{18} \mathrm{~F}$ ions, which emit $511 \mathrm{keV}$ annihilation photons. These photons are then detected with a camera, and particle position is triangulated. An example of the results obtained by Chaudhry [51] is shown in Figure 4.
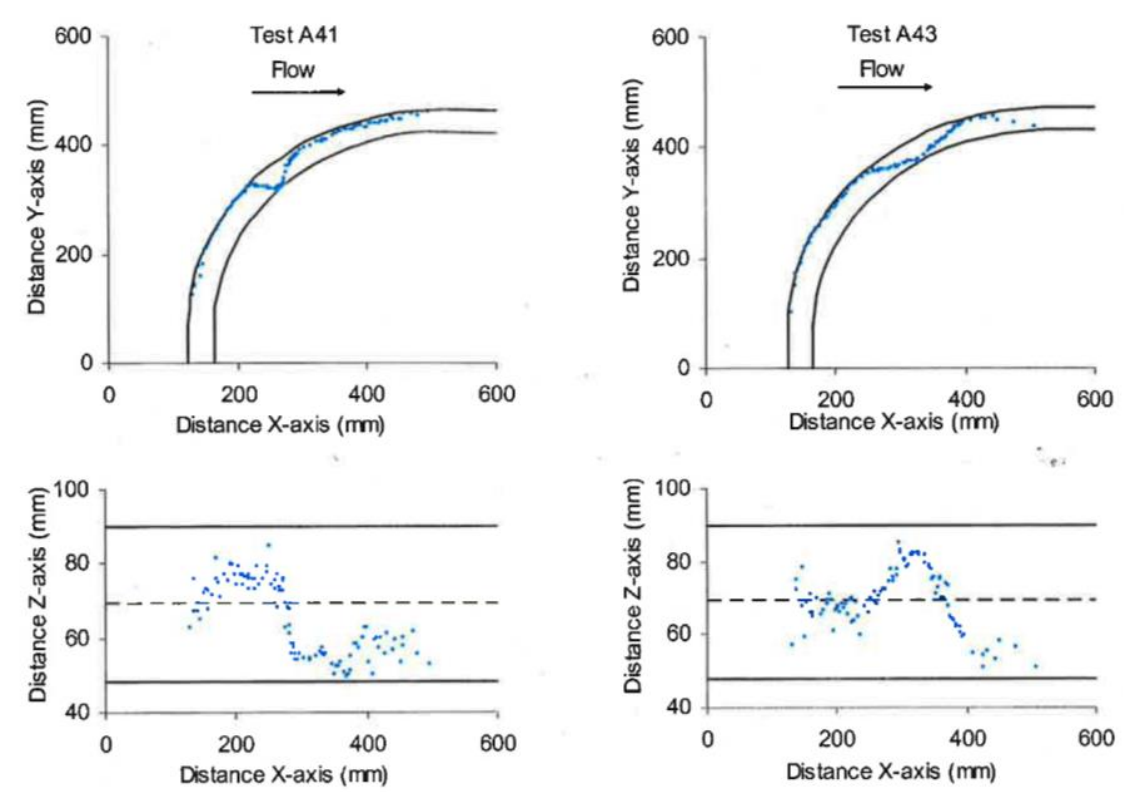

Figure 4: PEPT Results obtained by Chaudhry [51]

\subsubsection{The Concept of Particle Shielding}

Particle shielding is a concept that suggests pipeline wear decreases with increasing particle concentration in the pipeline $[36,53]$. High-velocity particle-wall impacts are partially mitigated by inter-particle contacts, whereby only a fraction of the conveyed particles come into direct contact with the pipe wall. Little is understood about the underlying mechanics of this phenomena, however previous studies by Andrews and Horsfield [54] and Bridle [22], demonstrated the variation that particle concentration had on the operating behaviour of the system. Andrews and Horsfield [54] analysed interparticle collisions from the perspective of wall erosion. While failing to reduce the number of particles striking the wall surface, an increase in interparticle collisions (achieved through increasing particle concentration) resulted in a wider distribution of impact velocities and impact angles. Bridle [22] found that by increasing the particle concentration in a single pneumatic 
conveying test bend, the percent fines measured was reduced. However the solids to air flow rates ( $\mathrm{kg}$ of solids per $\mathrm{m}^{3}$ of conveying air) encompassed dilute phase through to dense phase conveying types (more detail in Section 6.2). Further quantification of the influence of particle concentration on particle attrition is required in order to obtain a greater understanding of how lean phase pneumatic conveying systems operate, and whether the concept of particle shielding can be transferred to particle damage in addition to pipeline erosion.

\subsection{Concluding Remarks on the Process Factor}

It has been widely accepted that the majority of particle attrition occurring in lean phase pneumatic conveying pipelines can be attributed to contacts occurring at the bends. A number of geometrical and operating variables have been the subject of past studies, however the influence of particle concentration remains open for further exploration and understanding.

When aiming to control particle attrition, flow obstructions in the pipeline should be avoided, minimising the number of bends and using compression couplings. This applies specifically to the selection of coupling mechanism used to join the pipework. Such forms of mechanism include compression couplings and flanged pipes. Due to the variation in manufacturing quality and reliance on pipe cross section circularity, further analysis of this geometric factor shall not be undertaken. However, it should be noted that misalignment of the pipework is expected to lead to increased pressure drop and particle attrition.

The relationship between internal surface finish of the pipework, pipework material, and particle attrition has not been directly elucidated in the literature. However, it logically follows that by varying the surface finish, the frequency and magnitude of friction between the particle and wall will also vary. In this case, particulate attrition is expected to vary based on previous observations of shear load sensitivity. Additionally, the cross-sectional particle concentration within the pipeline have been shown to vary with respect to variation in pipe wall roughness $[26,27]$. Therefore the distribution of particles entering the bend is expected to vary with respect to pipe wall roughness. 


\section{Influence of Physical Particle Properties on Attrition Behaviour}

Particle failure is defined as the separation of a single particle into two or more child particles. The probability of a particle failing and the behaviour by which failure occurs are affected by mechanical and geometrical particle characteristics, dictating breakage behaviour. Further to this, the loading scenario to which the particle is subjected affects the mechanism of attrition observed.

\subsection{Particle Material Characteristics}

There is currently a lack in understanding in precisely how the material properties, in conjunction with applied loading conditions, affect attrition behaviour [55]. Key material properties and their influence on particle attrition behaviour are addressed here.

\subsubsection{Particle Strength}

Particle strength is the minimum amount of stress that may be applied to a particle to cause macroscopic damage to the particle structure.

\subsubsection{Tensile and compressive}

Tensile tests can be challenging to execute due to the difficulty in obtaining a suitably sized and shaped specimen appropriate for the test. There is also the case of secondary shear stresses generated in various methods of the tensile test which invoke secondary failure mechanisms in the material [56]. While Griffith's theory proposes that the ratio of compressive to tensile strength is equivalent to a value of 8 where tensile failure is the dominant failure mechanism [57], further experimentation has not supported such a simple relationship [58]. In addition to this, the strain rate is critically important for agglomerate materials in obtaining representative characteristics of material properties [3].

\subsubsection{Yield Stress and hardness}

Yield stress can be measured with a number of methods, of which indentation and compression are common. Indentation uses an indenter of known geometry to penetrate and inflict plastic deformation in the material surface. Such a test proves challenging on particulate materials where little surface area is available, requiring the use of the nanoindentation technique [59]. According to Bika et al., hardness is a function of tensile yield strength, elastic modulus, Poisson's ratio and total strain (depending on material behaviour) [60]. The subsequent conclusion drawn from these observations is that the hardness value is exclusive to the conditions under which the test was performed, and may not be used as a holistically defining material property. This extends to the Young's modulus or fracture toughness which may also be calculated from these measurements.

\subsubsection{Dynamic Yield Strength}

Especially in the case of wet agglomerates, viscous effects play a significant role in determining the particle deformation behaviour. These viscous effects are strain-rate dependant [61], therefore calling for a definition of yield strength that accounts for strain rate. The concept of dynamic yield strength is introduced by Hawkyard [62], to address this behaviour in the form of Equation (1) for a cylindrical specimen.

$$
\frac{1}{2} \rho U^{2}=Y\left[\frac{A_{0}}{A_{1}}-1+\ln \left(\frac{A_{1}}{A_{0}}\right)\right]
$$

Where $A_{0}$ is the initial contact area, $Y$ is the dynamic yield stress, $U$ is the impact velocity, $\rho$ is the particle density, and $A_{1}$ is the deformed (post-impact) contact area. Conversion between the results 
of dynamic measurement methods has been stated to be irreconcilable with the equivalent quasistatic method due to the significant difference in strain rate [3].

\subsubsection{Fracture toughness}

Defined as the critical crack driving force, fracture toughness is a measure of a material's ability to resist crack growth in the presence of an existing crack [63]. This property is typically measured through the flexural bending of notched bars, specified in ASTM E399-90 [64]. Preparation of very specific material geometries to ensure that a valid result is obtained; such geometries are unobtainable for many particulate materials. Furthermore, increased complexity is found in the testing of agglomerate materials in defining fracture area corrections accounting for sample porosity [60]. It has also been argued that the failure mechanism for porous agglomerates may not be due only to the failure of a single primary crack, but rather the simultaneous propagation of multiple fractures (for example, [65]).

Two approaches are well known in fracture mechanics to fundamentally explain crack propagation through a material. Griffith [57] proposed that specific surface energy should be considered as one of the material properties of primary importance, whereas Irwin [66] proposed that strain energy release rate is of primary importance, especially in ductile materials. More recently, Ouchiyama et al. [67] found that the fracture toughness cannot be exclusively used to fully describe the attrition behaviour of particulate materials.

\subsubsection{Particle Structure}

The microstructure of a particle dictates the distribution of defects or cavities, which along with the atomic structure, influence the location and orientation of fracture planes.

\subsubsection{Agglomerate Particles}

Agglomerates present a unique challenge in terms of understanding their fracture modes due to the lack of continuity in their structure. Reynolds et al. [3] define three different types of agglomerate structure; binderless describes an agglomerate whereby van der Waals or other similar micro-scale forces hold the constituent particles together, solid describes agglomerates where solid connections are in place between the constituent particles, and wet describes agglomerates containing liquid in the interstitial spaces between constituent particles.

Through acknowledgement of the complex and diverse structures of agglomerate materials, it may be appreciated that their attrition behaviours embody a number of distinct mechanisms. Such mechanisms have been the focus of a number of studies both experimental $[65,68]$ and simulated [69-73] in nature, some of which are summarised in Figure 5. 
(a)
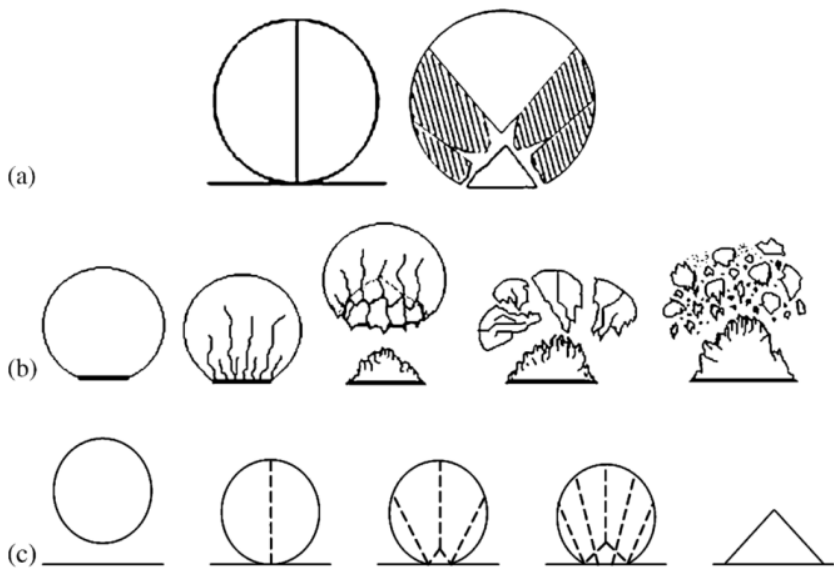

Figure 5: Agglomerate failure methods by impact as described by Reynolds et al. [3] and adapted from the work of Salman et al. [74], for (a) solid, (b) wet, and (c) binderless structures. Impact velocity increases from left to right.

\subsubsection{Crystalline Particles}

The production process by which crystalline materials are manufactured has been shown to have a significant influence on their attrition propensity [75]. This has been demonstrated not only with respect to the crystal drying mechanism selected, but also for subsequent handling processes within the manufacturing plant attributed to various applied loads on the particles. If a crystalline material is of a semi-brittle nature, it will typically fail via the previously described, chipping mechanism [76]. If the crystal fails via a pure brittle mechanism, rupture along preferred fracture planes is expected. The selection of the failure mechanism by which a crystal fails can be due to the distribution of loading forces across the topography of the particle (i.e. particle orientation with respect to impact plane).

In the case where a particle is composed of two or more crystals, cleavage may occur at the interface. It is worth noting that such a particle failure would yield child particles that are relatively undamaged [75]. This is important when considering the fatigue characteristics of particulate materials, and the formation of child particles in the form of whole crystals as opposed to platelets of significantly smaller mass.

\subsubsection{Pelletised Materials}

Pelletised materials demonstrate a wide range of attrition behaviours due to the extensive range of feed materials and manufacturing processes that are used. Thomas et al. [77-79] published a series of articles acknowledging these factors. Particular emphasis was given to the evaluation of pellet hardness and durability, along with the influences of feedstock and manufacturing processes on these qualities. Furthermore, pellets are generally required to give a specified nutritional content (in animal feeds) or calorific value (in combustion processes) adding another layer of physical requirements to the product. Such diversity in material composition and form inevitably leads to an equally diverse range of failure modes.

\subsubsection{Coefficient of restitution}

When considering elastic-plastic collisions in dynamic processes, the kinematic/kinetic/energetic and tangential coefficients of restitution should be accounted for. Furthermore, a work-hardening model is required if the material in question displays such behaviour, as this will alter the coefficient of restitution of subsequent impacts and the mode of kinetic energy dissipation [80]. If irregularly 
shaped particles are being considered, as is the case in the majority of existing processes, resulting rotational effects must be considered in the selection of the coefficient of restitution [81].

A number of studies consider almost fully plastic impacts [61]. Fu et al. [82] investigated the influence of various wet agglomerate material parameters on the coefficient of restitution and contact ratio observed across a range of impact velocities. Primary particle size, binder viscosity, binder ratio, and granulation time were all compared against impact velocity with respect to the measured coefficient of restitution. As these particles demonstrate adhesive elastic or elastoviscoplastic behaviour, it was found that a minimum of $97 \%$ of the impact kinetic energy is dissipated in intra-particle viscous flow at low impact velocities, or bulk viscoplastic flow at high impact velocities. This work is further supplemented by Mangwandi et al. [83] who looked at the coefficient of restitution of wet, melt and binderless agglomerates and proposed a model to account for strain-hardening.

\subsection{Particle Geometry}

Particle geometry refers to the physical profile of a particle, which influences a breakage propensity and resulting child particle size distribution.

\subsubsection{Particle Morphology}

Spherical particles have been the subject of numerous studies across a wide range of loading conditions $[68,69,72,82,84,85]$. This simplification the attrition process, as this particle geometry demonstrates no variation with orientation. It has been generally found that spherical particles can fail at low impact energies through the propagation of meridional cracks, producing a small number of large fragments. As the impact energy is increased, a greater number of fragments are produced [70], in addition to complete disintegration of the contact zone for agglomerate structures [65]. This relationship with applied force is also observed for double impact testing [86].

Cubic particle failure has been studied in some detail, with simulation work [87], and experimental work with materials such as salt, magnesium oxide and potassium chloride [88]. In terms of impact attrition, it has been shown that the orientation of impact is critical to the mode of attrition observed. If the impact occurs at one of the corners or edge of the particle, semi-brittle failure is observed due to a concentrated stress distribution. Ghadiri et al. [75] studied cubic $\mathrm{NaCl}$ particles where a 'full face' impact (a side of the cube impacts parallel to the wall surface) was observed. The particle was observed to unload elastically from the impact, and did not suffer any of the damage due to chipping, AS was observed when corners or edges of the particle form the contact surface.

Antonyuk et al. [89] studied the breakage behaviour of L-threonine crystals and $\mathrm{Al}_{2} \mathrm{O}_{3}$ agglomerates by impact and compression respectively. Both particle geometries were of cylindrical form, however, the aspect ratio of the crystalline material conformed to a needle shape. The compression experiments (applied force perpendicular to longitudinal axis) found that the agglomerates cracked longitudinally along the axis of the cylinder whereas the crystals typically result in two or three transverse cracks.

Other particle shapes such as orthorhombic [76], and generally random-shaped particles [90] have also been investigated on a qualitative basis, however the stress distribution becomes more complicated under these circumstances. Briesen [91] modelled crystal attrition with respect to impeller collisions and found that if a crystal geometry contains sharp corners, it is more likely to break than if it was spherical. 


\subsubsection{Particle Size}

It has been repeatedly demonstrated that particle strength decreases with increasing particle size $[75,85,88]$. Additionally, when repeated impacts have been considered, the rate of attrition across subsequent impacts increases with initial particle size [76]. These trends have been attributed to the likelihood of a greater number of imperfections in larger particles, which in turn provide a greater number of cracks which may propagate as a result of an imposed load [68].

This accepted, it has been demonstrated that even for particles of the same material composition, size and shape, a range of particle strengths are observed [92]. The size distribution of the child particles is commonly approximated by a Weibull distribution function $[14,68,93-98]$.

In the case of agglomerate primary particle size, it has been shown that a narrowing of the particle size distribution corresponds to a reduction in the agglomerate strength [99]. This is due to the inability of primary particles to layer themselves within agglomeration processes such as a high shear mixer. Generally a smaller primary particle size is associated with a higher agglomerate strength (in conjunction with other factors such as binder viscosity and agglomerate density) [100].

\subsection{Particle Fracture}

The particle failure mode captures the particle response to an applied load for the case when the particle strength (whether locally or globally within the particle) is exceeded. Particle fracture modes will be defined, followed by discussion of the fracture plane distribution and resulting distribution of particle fragments.

\subsubsection{Fracture modes}

Particulate failure modes have been broadly divided into three categories [65]; brittle failure where there is no significant plastic deformation, ductile failure characterised by extensive plastic deformation, and the transitional semi-brittle failure describes the occurrence of fractures initiated by plastic deformation [55].

The failure mode of a particulate material is subject to a wide range of physical factors. In the case of materials such as polymers, temperature of the particle is critical in determining the failure mode [101] with respect to the material-specific glass transition temperature. Agglomerate failure modes are heavily dependent on the binding mechanism, the properties and proportion of the binder, and in the case of wet binders, the interaction between the binder and the primary particles $[3,85]$.

\subsubsection{Fracture Plane Distribution}

The resulting size distribution of child particles can be described by one of two mechanisms: fragmentation and chipping. Both of these mechanisms correlate to different fracture planes within the particle body.

Particle fragmentation is the separation of a particle into two or more child particles of comparable size. This mechanism is the result of the formation and propagation of median, oblique or radial fissures throughout the entire volume of the particle [55].This is typically associated with highmagnitude loading conditions, whereby the stresses applied to the particle exceed the particle strength and generate free surface area via fracture planes.

Chipping or surface erosion, results in the removal of small fragments from the particle surface through the creation and propagation of sub-surface lateral cracks. These cracks are produced during the unloading phase of a particle impacting on a surface due to the production of residual tensile stresses [102]. The fragments produced as a result of this process are typically much smaller than the parent particle, and tend to originate from the corners or edges of the particle [55]. Ghadiri 
and Zhang $[55,88]$ developed and validated a mechanistic model to predict the rate of attrition of semi-brittle materials. $\mathrm{MgO}, \mathrm{NaCl}$ and $\mathrm{KCl}$ cuboid particles were prepared and impacted upon a hard surface. It was determined that the hardness of the target had little influence on the attrition results when the ratio of particle hardness to target hardness was less than, or equal to, unity. It was shown that attrition by impact (addressed in Section 5.4.1) did not follow a first-order relationship, attributed primarily to a work-hardening effect where particles become harder with subsequent impacts. This contrasts with previous work performed by Vervoorn and Austin [103], who had originally proposed that this attrition mechanism could be described as a first order process.

\subsection{Particle Attrition Mechanisms}

The loading conditions to which a particle is subjected, define the mode of failure and the size distribution of the resulting fragments. Shear, crush and impact loading, along with the influence of fatigue will be addressed. Focus is attributed to particle attrition due to impact as this research accounts for dilute phase pneumatic conveying, whereby the dominant source of attrition is expected to be caused by particle-wall impacts [104].

\subsubsection{Impact}

Particle failure by impact has been identified with processes such as lean phase pneumatic conveying [38], dependant on the type of particulate material under consideration [9]. Various aspects of this mechanism will be discussed in turn, followed by a survey of the current testing methods available.

\subsubsection{Impact Velocity}

Impact velocity has been repeatedly shown to have the greatest influence on the degree of particle attrition observed. With increasing impact velocity the impact energy increases, and hence is more likely to exceed the inherent strength of the particle. The mechanism by which particles fail with respect to impact velocity is fundamentally dependent on the particle material, which is why the probability of particle fracture and the subsequent size distribution of fragments can only be compared between particles of the same mechanical properties. An example of the influence of breakage behaviour of agglomerates was published by Subero and Ghadiri [65]. This behaviour was resolved into a map against the void characteristics of the particle (Figure 6). It can be seen that not only the breakage behaviour changes with increasing impact velocity, but also the frequency (and hence probability) of particle fragmentation. 


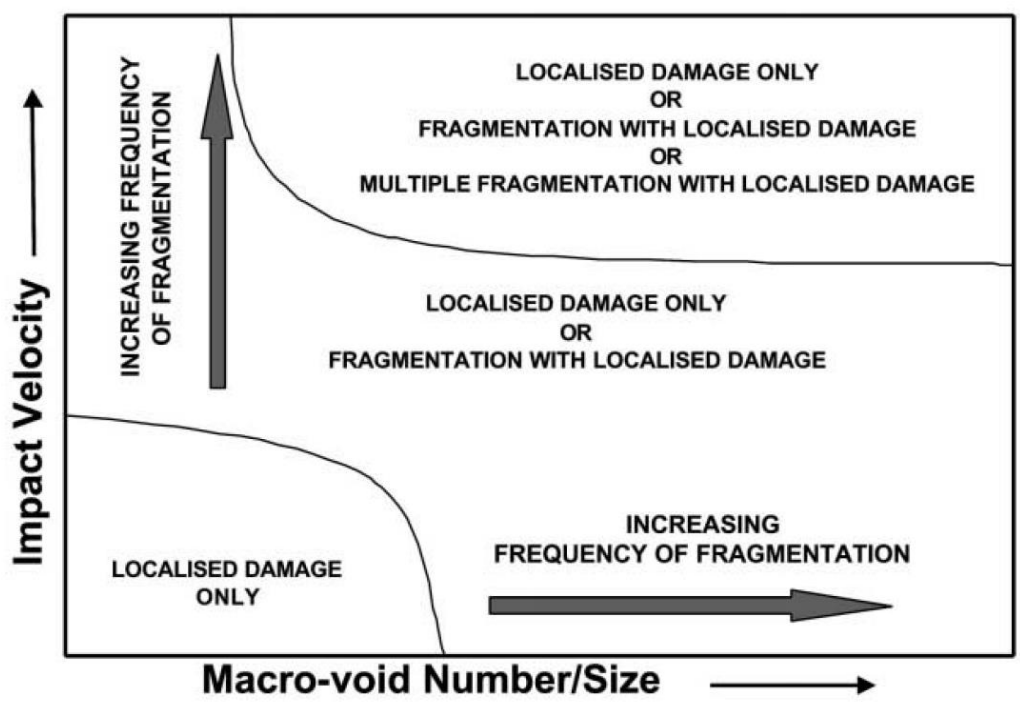

Figure 6: Agglomerate Breakage Behaviour Map as presented by Subero and Ghadiri [65]

To further illustrate the variable response of materials Zhang and Ghadiri [88] demonstrated that the attrition propensity of three different crystalline materials could be approximated with a power law relationship - with the indices unique to each respective material. Furthermore, these indices changed with subsequent impacts due to the work-hardening behaviour of the crystals.

It is therefore apparent, that while in the vast majority of cases, particle attrition increases with increasing impact velocity, the response of the particle is specific to the physical properties of the particle.

\subsubsection{Impact Angle}

The impact angle of a particle against a surface is here defined by the angle subtending the particle velocity vector and the plane represented by the material surface.

It has been shown that the severity of particle attrition increased as the impact angle increased towards a normal impact scenario. This is due to the relatively large normal force vector in comparison with the tangential force vector. An idealistic study was conducted by Cheong et al. [105] looking at the attrition behaviour of glass spheres. It was concluded that the mean size of the fragment size distribution reduced with increasing impact velocity. The fragmentation regime and its relationship with respect to the angle of impact are summarised in Figure 7. A similar regime is given by Laarhoven et al. [106], shown in Figure 8.

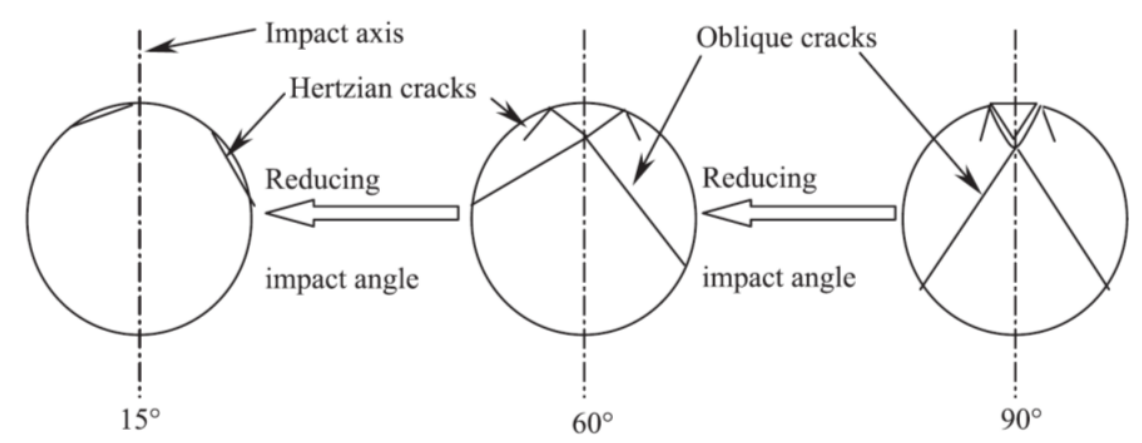


Figure 7: The influence of impact angle on the breakage mechanism of glass spheres as reported by Cheong et al. [105]

\begin{tabular}{l|l|l}
\hline Magnitude & \multicolumn{2}{|c}{ Direction } \\
\hline "Wear" & Normal & Tangential \\
\hline Low force & & \\
"Figh force & & \\
"Fracture" & & \\
\hline
\end{tabular}

Figure 8: Influence of Impact Angle and Impact Force as Reported by Laarhoven et al.[106]

The influence of impact angle on soft agglomerate structures was studied by Samimi et al. [107]. In contrast to the commonly obtained result, it was found that these agglomerates suffered greater damage as the impact angle was reduced. It was concluded that this was due to the ductile nature of the materials, which were more sensitive to attrition by a shearing mechanism.

When studied numerically through the application of Discrete Element Method simulations, Moreno et al. [108] reported that the observed damage ratio increased with respect to both impact angle and impact velocity. The simulation contained particles representative of glass beads (in the form of an isotropic, spherical agglomerate) and a wall surface representative of steel. The damage ratio was defined as the ratio of broken interparticle contacts to the initial number of interparticle contacts within the unbroken particle. When the normal component of the impact velocity was held constant, the damage ratio was approximately constant, regardless of the impact angle. Furthermore, the number of fragments are reduced with reducing impact angle. Overall the magnitude of the normal velocity vector was found to be the most influential, with the tangential velocity vector influencing the size distribution of the child fragments. These findings were later supported experimentally by Samimi et al. [107], who also confirmed the importance of the particle mechanical response in the determination of the breakage characteristics, and sensitivity to tangential impact velocities.

Namely, materials failing in a ductile regime, will be more sensitive to the shear loading experienced at shallow impact angles.

\subsubsection{Strain Rate}

Impact and quasi-static compression experiments have been used to compare the effects of strain rate on particle failure and crack propagation. Particles are typically well-defined in such studies, taking the form of spherical agglomerates or isotropic solids. Cheong et al. [105] conducted such an investigation into the breakage behaviour of glass spheres. The analysis of the results proceeded by equating critical loads required for initiation of a Hertzian cone crack. It was concluded that the impact velocity required to initiate a Hertzian crack (pictured in Figure 7) can be reasonably predicted through slow compression testing. According to Subero-Couroyer et al [109], Couroyer [110] had earlier found that for ceramic particles failing via a brittle mechanism, the breakage characteristic observed through impact could be correlated to quasi-static crushing. 
The work of Schönert [101] also found that spheres and discs made of glass and poly(methyl methacrylate) (PMMA) exhibit the same failure scheme under both impact and compression loading. However, the strain rate was concluded to influence the material flow during loading, and therefore influence the stress field within the particle and the resulting crack pattern.

Chau et al. [111] compared compression tests performed under quasi-static and dynamic conditions with respect to the failure of plaster spheres of various concentrations. In the dynamic series of tests, the impact velocity varied between 1.349 to $2.485 \mathrm{~m} / \mathrm{s}$. The key finding from this study was that the contact force was larger in the quasi-static case, and the energy required for fracture was larger in the dynamic case.

The variation of material properties with respect to strain rate has been investigated through the application of microindentation testing of salt particles [75]. Absolute values of microhardness are dependent on the strain rate imposed by the indenter. This is due to the presence of material behaviours such as creep caused by a viscoplastic response. In this context, it is recommended to specify a strain rate across which materials can be quantitatively compared. This observation demonstrates that material failure can be sensitive to loading strain rate, and not only the magnitude of maximum load alone. This concept is supported by Reynolds et al. [3] and Iveson et al. [112], who explicitly state that the breakage of agglomerate particles are strain-rate dependant within the granulation context. Furthermore, Gorham et al. [113] concluded that PMMA spheres are sensitive to the strain rate caused by an applied load, and this therefore dictates the particle failure mechanism.

In summary, the influence and significance of strain rate on the attrition mechanism for a given particle is fundamentally dependant on the particle material and the failure mode that is characteristic of that material.

\subsubsection{Shear}

Shear forces in lean phase pneumatic conveying are primarily attributed to particles sliding along the pipe around bends and the lower surface on straight sections. While this form of particle attrition is not expected to find direct relevance to lean phase pneumatic conveying systems, it is included here for completeness.

Ghadiri et al. [114] performed a number of shear experiments on porous silica catalyst carrier beads (spherical) at low normal stresses ( 25 and $50 \mathrm{kPa}$ ) with respect to attrition evolution within an annular shear cell (see Figure 9). The theories of Ouwerkerk [115] and Neil and Bridgwater [116] were evaluated and modified, showing a good fit to high normal stresses, yet less-than-adequate fitting to low normal stress results. A potential reason for this observation was attributed to the presence of particle breakage and subsequent generation of smaller particles through the fragmentation process. With respect to the magnitude of fines generated by surface wear, this was deemed to increase constantly with the strain rate. It is worth noting that experiments using the annular shear cell stress particulate materials at very low strain rates - in the order of 1 RPM or 6.8 $\mathrm{mm} / \mathrm{s}$. Such strain rates may struggle to find similarity with the conditions within lean phase pneumatic conveying systems, where particle velocities are of the order of $25 \mathrm{~m} / \mathrm{s}$. Furthermore, it was suggested that the presence of fine particles in conjunction with coarse particles may reduce stress localisation within a particle bed, therefore reducing the overall rate of attrition. 


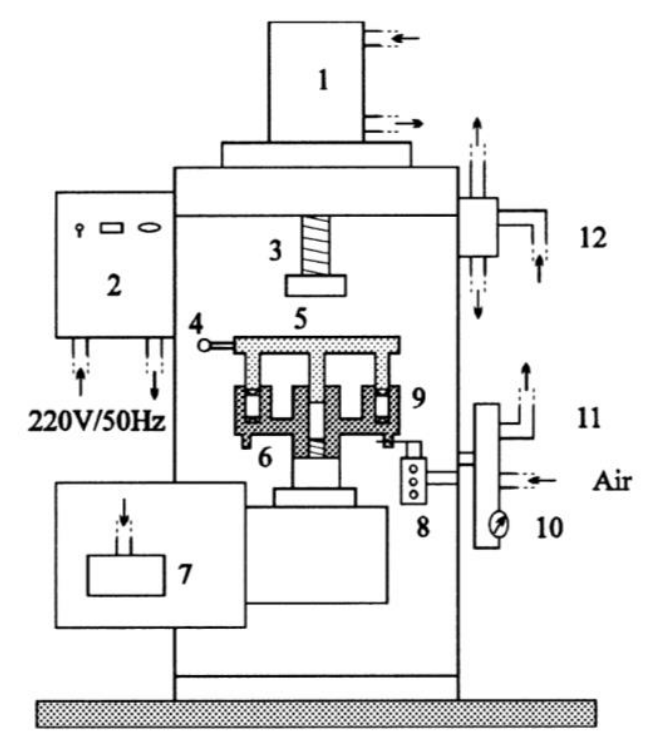

Figure 9: Annular Shear Cell as used by Ghadiri et al. [114]

Hare et al. [117] considered the attrition of pharmaceutical powders during the crystallisation process within agitated driers. The key findings from this work were the determination of relationships between the bed height and the relative strain rates imposed by an impeller. These results were then compared with experiments performed in an annular shear cell. The difference in relative velocities may be sufficient to cause a shearing effect in a lean phase pneumatic conveying system. Whether the bed thickness reaches a sufficient depth around the bends in the pipe, remains to be seen.

As alluded to in the preceding section on attrition as a function of impact angle, sensitivity to shear stresses was used to explain the increasing particle attrition with decreasing impact angle. This was typical of materials failing predominantly through a ductile mechanism [107].

\subsubsection{Crushing}

Crushing is the application of a compressive load to a particle under quasi-static conditions. Quasistatic compression tests typically take a physical form similar to the Brazil Test, whereby two surfaces are brought together at low speed in order to apply a load to a specimen. This test methodology is the subject of a number of standards, particularly with respect to the analysis of catalytic particles such as in ASTM D6175 [118].

This approach to evaluating the particle strength of a given material is appropriate only to welldefined, regular particle geometries such as spheres or cylinders, as the crushing stress can be resolved [109]. Irregular particle shapes prove challenging for this test methodology as numerical methods are required to resolve the complex stress analysis or analytical considerations as in the case of Aman et al. [90].

Quasi-static compression loading using $12.7 \mathrm{~mm}$ diameter acrylic spheres has been conducted by Chaudhri [119], who described the development of stresses within the sphere and subsequent fracture patterns. Similar experiments were performed by Wu et al. [84] with 50,60, and $75 \mathrm{~mm}$ diameter plaster spheres, Gorham et al. [113] with $8 \mathrm{~mm}$ diameter PMMA spheres, and Antonyuk et al. [68] using three types of spherical granulate materials $\left(\gamma-\mathrm{Al}_{2} \mathrm{O}_{3}\right.$, Köstrolith, and sodium benzoate). 
Each material exhibited prominent meridional cracking, however the exact geometry of the fissures and the proportion of smaller fragments produced were specific to both the particle material and loading conditions.

The material used to form the surfaces applying the load to the particle is of equal importance in quasi-static testing. This was investigated by Shipway and Hutchings [120] who tested a range of surface materials broadly categorised into plastically deforming and elastically deforming behaviours. When a plastic surface was used to apply load, particle failure was initiated at the surface of the particle, whereas the use of elastic surfaces results in internal failure initiation of the particles by shear forces.

\subsubsection{Fatigue}

Fatigue is a phenomenon whereby particles undergo repetitive loading, and as a result, suffer strength reduction and catastrophic failure.

Goder et al. [121,122] looked at a bed of narrow size range of particles, subjected to repeated compressions applied by a platen. The materials under scrutiny were classified as; brittle, high strength granulated, and ductile materials. Fatigue curves were generated for each of these materials with respect to the percentage of material passing through a specified sieve. Particle damage was generally found to increase with increased loading magnitude and number of compressive cycles. The ductile material was found to agglomerate under conditions of high compressive stress.

Through modification of Griffith's Theory of crack propagation, Han et al. [123,124] proposed a model for prediction of particle failure by repeated compressive loading. The ratio of fatigue compressive strength $\left(\mathrm{P}_{m}{ }^{\prime}\right)$ of a particle to the compressive strength $\left(\mathrm{P}_{\mathrm{m}}\right)$ of the original particle was defined as in Equation (2); where $\sigma^{*}$ and $\mathrm{P}^{*}$ are dimensionless numbers, $\mathrm{N}$ is the number of compression cycles, and $\mathrm{P}_{1}, \mathrm{P}_{2}$, and $\mathrm{P}_{3}$ are dimensionless groups whose definition may be found in Han et al. [123]. The results of this analysis correlated well with $\mathrm{NaCl}$ and $\mathrm{MgO}$ crystals, and with granulated fertiliser particles. In a later publication, Han et al. [125] extend this theory to impact loading with deviations of up to $25 \%$.

$$
\frac{P_{m}^{\prime}}{P_{m}}=\sigma^{*^{3}}=\left\{1+\frac{P_{1}}{\exp \left[P_{3}-P_{2}\left(P^{*}\right)^{1 / 3}\right]^{2}}\right\}^{-3 / 2 N}
$$

This model was later modified by Rozenblat et al. [126] in order to discretise for loading magnitude. In order to achieve this, the calculation method was modified to define particle strength in terms of crushing force instead of stress. Application of this model to experimental results showed good correlation, however only Dead Sea salt crystals were considered.

A broad study looking at the fatigue failure of the same three materials used by Han et al. [123], was reported by Kalman et al. [127] across four different impact tests. Fatigue curves were presented and correlation across each of the impact methods was demonstrated. General trends in the results supported the vast majority of current research in that particle damage increases with increasing impact velocity and increasing the number of impact cycles.

Tavares and King [128] applied damage mechanics concepts to particle fatigue by impact loading. Through the use of the Ultrafast Load Cell (UFLC) impact tester, they developed Equation (3), which describes the amount of damage sustained $\left(D_{n}{ }^{*}\right)$ by the particle in the $n$th impact; where $\gamma$ is the 
damage accumulation constant, $E_{k, n}$ is the kinetic energy of the striker at the $n$th impact per unit mass of the particle, and $E_{n-1}$ is the energy required to completely disintegrate the particle per unit mass of the particle in the previous event. This method requires the calibration of a single factor, $\gamma$, from experimental data and found excellent correlation with experimental results.

$$
D_{n}^{*}=\left[\frac{2 \gamma}{\left(2 \gamma-5 D_{n}^{*}+5\right)} \frac{E_{k, n}}{E_{n-1}}\right]^{\frac{2 \gamma}{5}}
$$

In a further study Tavares [129] resolves the significance of the only empirical factor in the model, $\gamma$, to be independent of particle shape, and weakly dependant on particle size within the range tested. Additionally, the model was successfully applied to results obtained from gas-blast apparatus testing, and therefore not specific to the particle stressing mode.

\subsection{Experimental Techniques for Measuring Particle Attrition}

All experimental tests with respect to particle attrition may be broadly categorised into one of two groups: single-particle, and multi-particle tests. Single particle tests enable the study of particle behaviour under closely controlled, well-defined conditions in order to investigate the fundamental particle behaviour. The drawback of single-particle testing, is that the test conditions are often difficult to correlate with the conditions present in industrial processes. Multi-particle experimentation is the vice-versa of this situation. While the loading conditions experienced by each particle are not known (discounting numerical simulations), the conditions the material is subjected to can be more easily correlated with industrial processes.

The experimental approach specific to impact testing can be broadly grouped into two areas;

Firing a particle at a rigid surface and include:

1. Gas-Blast Tester: See Section 5.5.1 for description.

2. Centrifugal Accelerator: See Section 5.5.2 for description.

3. Drop Test: particles are allowed to fall under the influence of gravity to impact on a surface or bed of material.

4. Rotating Arm Test: A particle stream is dropped under the influence of gravity into the path of an array of targets attached to a rotating disc.

5. Tumbling Box Test: A sample of particles are placed in a box, which is then closed and rotated about a single axis.

6. Oscillating Box: Particles are placed in a box which is oscillated along its vertical axis.

Crushing a particle between a hammer and anvil made from a rigid material and include:

7. Resonate Cantilever Impactor: An oscillating cantilevered beam supports a particlecontaining cavity at its end.

8. Swing Pendulum Tester: See Section 5.5.4.1 for description.

9. Double Impact Tester: See Section 5.5.4.2 for description.

Methods 3, 4, 5, 6, and 7 involve either bespoke pieces of equipment or significant particle interaction throughout the application of loading forces, and therefore shall not be further addressed in this section. As the particle-wall impact mechanism is expected to dominate in lean phase pneumatic conveying systems, test methods without secondary applied loads (such as those applied by other particles) shall be further examined. 


\subsubsection{Gas Blast Tester}

The Gas Blast Tester consists of a compressed air flow to which particles are introduced and accelerated along a straight channel. Upon exit of this channel, the particle impacts a hard surface placed in the air stream. This test method is the subject of ASTM G76-13 [130] and was that of DIN 50332 (Withdrawn) [131] which offer guidance on the methodology of the test. As illustrated by Figure 10, many arrangements can be utilised in terms of mixing the particulate and gas flows, and extracting impact data.

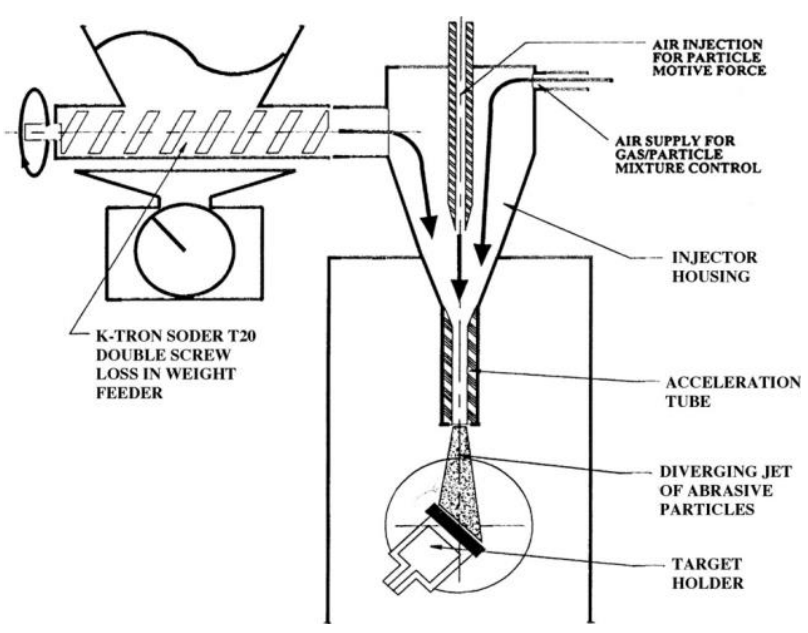

(a)

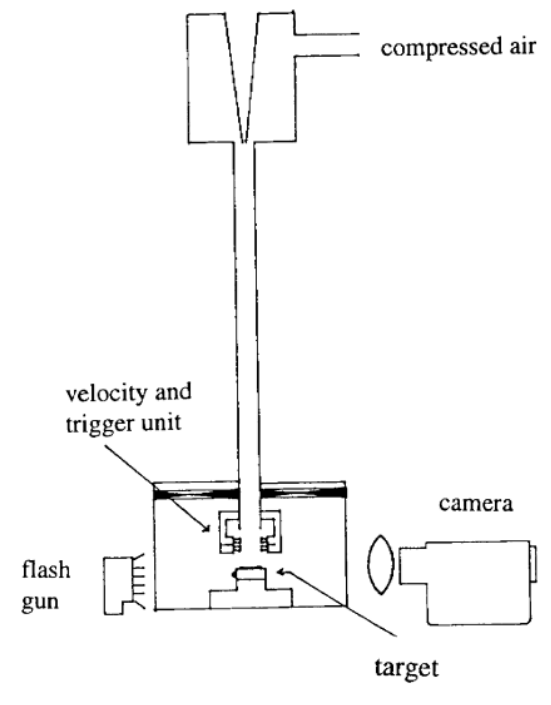

(b)

Figure 10: Gas Blast Tester as Investigated by: (a) Deng et al. [132]; (b) Zhang and Ghadiri [88]

Shipway and Hutchings [133] investigated the influence of the roughness of the air-particle outlet nozzle on the impact conditions within a gas-blast erosion tester. They found that the roughness of the nozzle bore had a significant influence on the velocity and trajectory of the ejected particles. The characteristics of this analysis are also dependant on the nature of the ejected particles. Generally, the rougher the nozzle bore, the lower the ejected particle velocity and wider the resultant particle stream, for a given air flow rate. Another study by the same authors looked at the influence of the target material [120]. Ductile and elastic target materials were found to yield different crack initiation locations within lead glass spheres of $(700 \pm 50) \mu \mathrm{m}$.

\subsubsection{Centrifugal Accelerator}

The centrifugal accelerator apparatus has been widely used for erosion studies and was the subject of the former Soviet Union Standard, GOST 23.201-78 [134]. This apparatus has more recently been used for the evaluation of particle attrition by impact [135] and comprised a rotating disc that contained a number of radial tubes. The disc was surrounded by an array of targets. The particulate samples were fed into the centre of the rotating disc and radially accelerated via the tubes and impacted against the target array. Figure 11 shows a schematic of such an apparatus. 

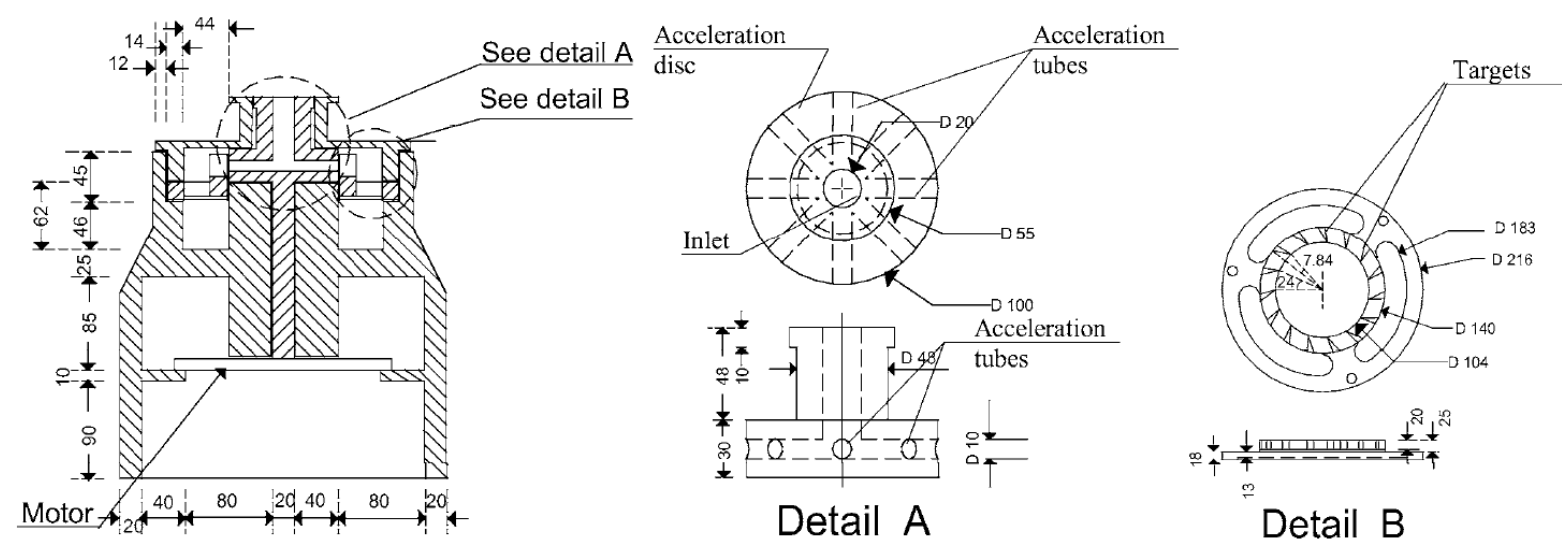

Figure 11: Centrifugal Impact Tester as Investigated by Chapelle et al. [136]

Burnett et al. [137] analysed the results obtained from a centrifugal accelerator test apparatus and the subsequent variations within are discussed in depth within the context of erosion. Here, the effect of particle spin was addressed, imparted by the method of acceleration within the test device. Angling the targets about the horizontal axis was suggested as a method in which to reduce the effects of particle spin on the impact (and hence particle loading) mechanics. Deng et al. [30], modelled the particle velocity vector at the outlet of the disc tube. The particle shape was found to influence the particle's tendency to spin on ejection from the disc tube, and below a specified shape angle, pure sliding was expected along the tube wall. The effects due to aerodynamic drag were deemed negligible.

\subsubsection{Comparison of Impact Testers}

A comparison of the gas blast tester and the centrifugal accelerator tester was conducted by Burnett et al. [138]. Particulate materials included two ceramics, one metallic, one elastomeric and one polymeric material, representing a wide range of material categories and behaviours with respect to erosion of the target material. With regard to the operational capacity of each apparatus (the operational parameters relevant for use in particle attrition studies), the conclusions of this study are summarised.

With regard to the gas blast tester:

- The identification of steady-state operation and particle impact velocity are difficult to define

- The range of particle concentrations that can be a achieved are limited

- Greater particle stream divergence is observed in comparison to the centrifugal tester

With regard to the centrifugal tester:

- Determination of particle impact velocity is straightforward

- Particle-gas interactions are lower than those of the gas-blast tester

- Steady state conditions are more readily defined

\subsubsection{Static Particle Tests}

The kinetic tests (Methods 7 and 8 ) where a hammer impacts a static particle at a specified velocity, have been widely studied (see Table 1, Section 5.5.5). The quasi-static form of the test is addressed in Section 5.4.3. Figure 12a and $b$ show examples of the Swing Pendulum Test and the Double Impact Test respectively. 


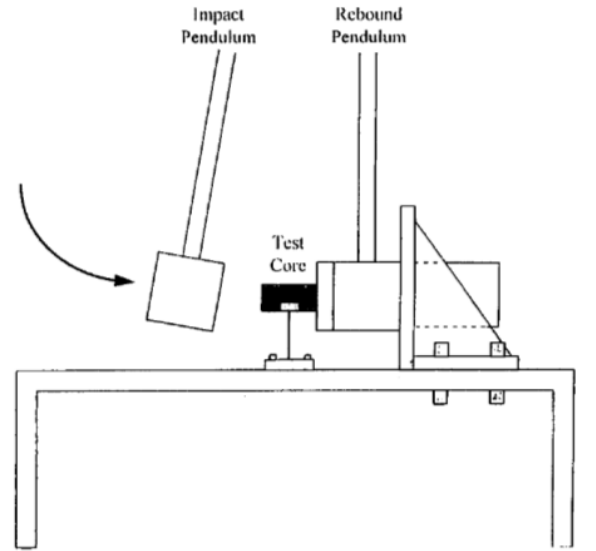

(a)

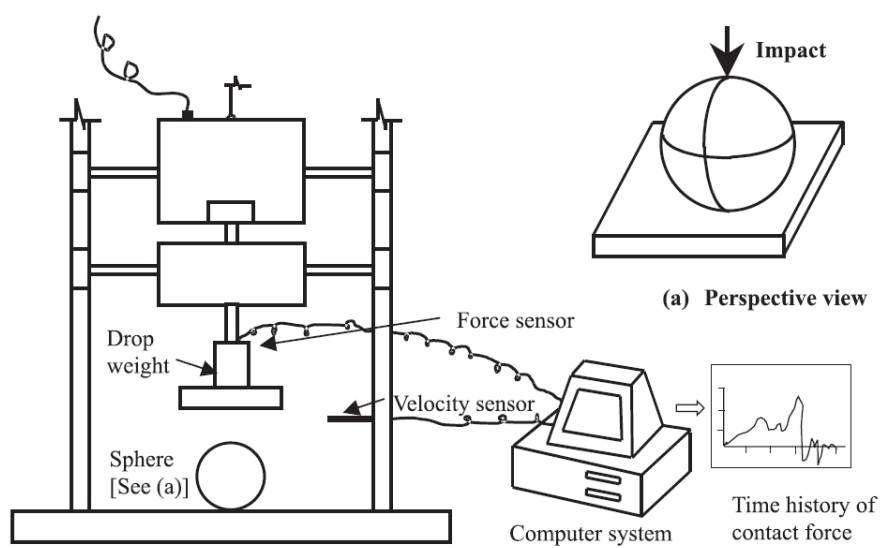

(b)

Figure 12: Examples of the Swing Pendulum Tester [139] and the Double Impact Tester [111]

\subsubsection{Swing Pendulum Tester}

Weedon and Wilson [140], and Sahoo et al. [141] utilised a twin pendulum apparatus to evaluate the degradation of iron ore and coal respectively. In both cases a spherical hammer was used to degrade particles, and the former of these two studies proposes a semi-empirical model to predict fines generation as a result of dropping material from heights during handling processes. A further publication from Sahoo et al [142] modifies this model for application to the prior study on coal. Upon inspection of the approach undertaken by these studies, it is evident that a significant number of particles must be studied in order to define the material-specific model best suited to the material. Furthermore, the methodology demands particles of sufficient size with respect to the measurement resolution in order for the test to be successful. The pendulum test is, however, capable of measuring the energy associated with particle breakage [143].

\subsubsection{Double Impact Test}

Krogh [144] investigated the influence of the impact velocity of the hammer dropped onto the particle sample within a double-impact tester. The analysis progressed by defined by three attrition functions:

- Crushing probability function

- Energy function

- Breakage function

These three functions account of the strength distribution of the particle sample, the strength of the particles with respect to their size, and the subsequent size distribution of the resulting fragments respectively. It was found that the velocity of the hammer did not noticeably influence the attrition behaviour of the particle; rather the impact energy (modified by changing the mass of the hammer) was determined to be the defining variable.

Tavares and King have published a number of works in which the data collected from a modified double impact test was used [128,145-147]. The Ultrafast Load Cell (UFLC) (see Figure 13), was a hybrid design between a double impact tester and a split Hopkinson Bar, capable of measuring the applied force to a resolution of $0.2 \mathrm{~N}$, with a time step of $100 \mathrm{~ns}$. Evaluation of particle fracture 
energy and the influence of material structure was achieved. From a fundamental fracture mechanics perspective, this apparatus is potentially the best equipped for particle failure analysis. However, the requirement of testing particles individually limits the size range of interest and may not conform to user time constraints.

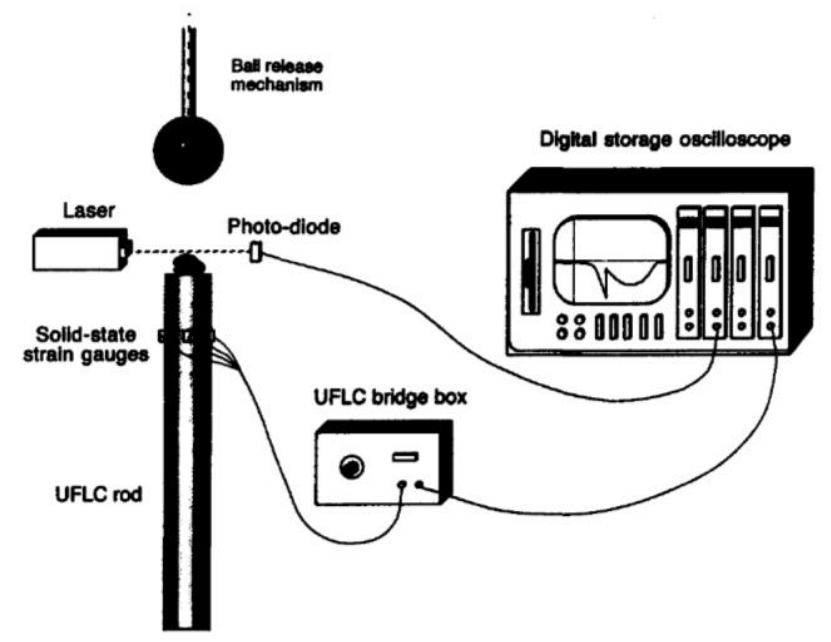

Figure 13 :The Ultrafast Load Cell [146] 


\subsubsection{Summary of Particle Attrition Testers}

Table 1 presents a summary of each of the particle attrition testers considered in this review. While each of the testers referenced from other works were not necessarily considered within the context of lean phase pneumatic conveying systems, the entire range is included for completeness and quick comparison for the reader.

Table 1: Summary of Particle Attrition Testers

\begin{tabular}{|c|c|c|c|c|c|c|c|}
\hline \multirow[b]{2}{*}{ Tester } & \multicolumn{5}{|c|}{ Operating Variables } & \multirow[b]{2}{*}{ Notes } & \multirow[b]{2}{*}{ Studies Used } \\
\hline & $\begin{array}{c}\text { Impact } \\
\text { Velocity/Strain } \\
\text { Rate Control }\end{array}$ & $\begin{array}{l}\text { Impact } \\
\text { Angle } \\
\text { Control }\end{array}$ & $\begin{array}{c}\text { Level of } \\
\text { Inter- } \\
\text { Particle } \\
\text { Interaction }\end{array}$ & $\begin{array}{l}\text { Secondary } \\
\text { Impact } \\
\text { Velocity }\end{array}$ & $\begin{array}{l}\text { Feed } \\
\text { Rate } \\
\text { Control }\end{array}$ & & \\
\hline $\begin{array}{l}\text { Gas Blast } \\
\text { Tester }\end{array}$ & $* *$ & $* * *$ & Low & Medium & $* * *$ & $\begin{array}{l}\text { Variations in velocity profile are } \\
\text { difficult to closely control. } \\
\text { Particle stream divergence } \\
\text { greater than centrifugal } \\
\text { accelerator. }\end{array}$ & {$[85,98,104,107,148$} \\
\hline $\begin{array}{l}\text { Centrifugal } \\
\text { Accelerator }\end{array}$ & $* * *$ & $* * *$ & Low & Medium & $* * *$ & $\begin{array}{c}\text { Close control over impact } \\
\text { velocity and impact angle. } \\
\text { Secondary impacts require } \\
\text { mitigation. } \\
\end{array}$ & {$[136,149,150]$} \\
\hline Drop Test & $*$ & $* * *$ & Variable & Low & $* * *$ & $\begin{array}{c}\text { Impact velocity limited to } \\
\text { particle terminal velocity. } \\
\text { Considerable head-room } \\
\text { requirement. }\end{array}$ & [151] \\
\hline $\begin{array}{l}\text { Rotating } \\
\text { Arm Test }\end{array}$ & $* *$ & $* * *$ & Low & High & $* * *$ & $\begin{array}{l}\text { Particles are accelerated by } \\
\text { impact with the target requiring } \\
\text { careful consideration of how to } \\
\text { mitigate secondary impacts. }\end{array}$ & [152] \\
\hline $\begin{array}{l}\text { Tumbling } \\
\text { Box Test }\end{array}$ & $*$ & - & High & - & - & $\begin{array}{l}\text { Low impact velocities coupled } \\
\text { with limited control of impact } \\
\text { conditions. }\end{array}$ & [153] \\
\hline $\begin{array}{l}\text { Oscillating } \\
\text { Box Test }\end{array}$ & * & - & High & - & - & $\begin{array}{l}\text { Low impact velocities coupled } \\
\text { with limited control of impact }\end{array}$ & [106] \\
\hline
\end{tabular}




\begin{tabular}{|c|c|c|c|c|c|c|c|}
\hline & & & & & & $\begin{array}{l}\text { conditions, however has the } \\
\text { potential to inflict more impacts } \\
\text { normal to the wall than the } \\
\text { tumbling box test. }\end{array}$ & \\
\hline $\begin{array}{l}\text { Resonate } \\
\text { Cantilever } \\
\text { Impactor }\end{array}$ & $* * *$ & - & High & - & - & $\begin{array}{l}\text { Limited control of impact } \\
\text { conditions. Offers the ability to } \\
\text { apply a large number of particle } \\
\text { loading events in a relatively } \\
\text { short amount of time. }\end{array}$ & {$[154]$} \\
\hline $\begin{array}{l}\text { Swing } \\
\text { Pendulum } \\
\text { Tester }\end{array}$ & $* * *$ & - & None & - & - & $\begin{array}{l}\text { Single particle test that requires } \\
\text { a significant number of } \\
\text { experiments in order to } \\
\text { characterise a material. Close } \\
\text { control of loading condition. }\end{array}$ & [139] \\
\hline $\begin{array}{l}\text { Double } \\
\text { Impact } \\
\text { Tester }\end{array}$ & $* * *$ & - & None & - & - & $\begin{array}{l}\text { Single particle test that requires } \\
\text { a significant number of } \\
\text { experiments in order to } \\
\text { characterise a material. Close } \\
\text { control of loading condition. }\end{array}$ & {$[84,129,144]$} \\
\hline $\begin{array}{l}\text { Annular } \\
\text { Shear Cell }\end{array}$ & $* * *$ & - & High & - & - & $\begin{array}{l}\text { Applicable only for the } \\
\text { characterisation of particle } \\
\text { failure under shear loading } \\
\text { conditions. }\end{array}$ & [114-117] \\
\hline $\begin{array}{l}\text { Quasi } \\
\text { Static } \\
\text { Crushing }\end{array}$ & $* * *$ & - & None & - & - & $\begin{array}{l}\text { Single particle test that requires } \\
\text { a significant number of } \\
\text { experiments in order to } \\
\text { characterise a material. Close } \\
\text { control of loading condition. }\end{array}$ & {$[68,84,90,109,113,119,120]$} \\
\hline
\end{tabular}

\section{** Medium Level of Control}

* Low Level of Control 
Not Applicable 


\subsection{Concluding Remarks on the Material Factor}

Some conflicts exist in the research. One such conflict is with respect to the influence of strain rate on particle attrition behaviour. This may, in part, be attributed to the conditions specific to each study. For isotropic materials failing in a brittle manner, it may be stated with reasonable confidence that the results between quasi-static compression testing and impact testing at low velocity are comparable. Once the impact velocity exceeds that of the elastic wave speed of the material, then this statement loses its applicability [120]. It is therefore important that within the context of lean phase pneumatic conveying, that this relationship is fully explored.

As has been demonstrated throughout the literature, the combination of particulate material, particulate structure and subjected loading conditions dictate the form of particle failure exhibited. These failure mechanisms are diverse in nature, and result in a variety of particle size distributions specific to each mechanism. When considering particle attrition in lean phase pneumatic conveying systems, it is important to consider the full spectrum of particle properties and loading scenarios. As has been demonstrated, different materials are more sensitive to specific loading scenarios, and any approach adopted in quantifying this behaviour requires sufficient flexibility to account for these. It is therefore imperative that experimental attrition testing be completed for each material whose behaviour is to be described.

When selecting a test method by which to characterise particle attrition behaviour, it is vital that the method be representative of the scenario for which the attrition behaviour is to be explain. For example, within lean phase pneumatic conveying systems, particles should be tested within a highvelocity impact regime, with the potential to supplement this information with other testing approaches. It should be noted that many methods of particle characterisation have been presented in the literature, however little standardisation has been adopted with respect to tester geometry or the associated attrition indices [121]. 


\section{Modelling of Particle Attrition in Lean Phase Pneumatic Conveying}

Studies conducted specifically into particle attrition within pneumatic conveying systems will be reviewed in this section. Works are arranged primarily by the nature of the work (whether derived from first principles, or through empirical modelling), and then chronologically by research group.

\subsection{Numerical Modelling Approaches}

Modelling approaches which draw on fundamental physical mechanics in order to describe the particulate flow and subsequent occurrence of particle attrition will be reviewed here. Attention shall be given primarily to the particle breakage model used.

Han et al. [155]

Han et al. [155] applied a two-dimensional Discrete Element Method (DEM) coupled with Computational Fluid Dynamics (CFD) model to simulate the results published by Bell et al. [156]. The Navier-Stokes equations were used in conjunction with the models presented by Papadopulos et al. [157], to describe the air flow and particle attrition behaviour respectively. A number of simplifying assumptions were also made: idealisation of particle shape to spherical geometry, particles smaller than $0.1 \mathrm{~mm}$ are disregarded, and two dimensional flow. Dilute phase pneumatic conveying was confirmed with a void fraction of 0.8 or higher. The simulations were conducted within a 2D channel: three straight sections $(12,4.8$, and $9.6 \mathrm{~m}$ respectively) connected by $0.35 \mathrm{~m}$ radius bends with a height of $8 \mathrm{~cm}$. Solids loading ratios of 1 and 4.5 were used. The reduction ratio describes the ratio of the surface area of the particles before and after the test and was the attrition criteria used. The simulated results were comparable to the experimental data of Bell et al., however, underprediction of particle attrition was observed with increasing numbers of passes through the system. This was attributed to the lack of a fatigue function within the breakage calculations, and use of different initial particle size distributions. Increasing the solids loading ratio was found to reduce particle attrition on a qualitative basis.

Hanley [158] and Hanley et al. [6,94,159]

The doctoral work presented by Hanley [158] produced two models to describe particle attrition: the first was a discrete element model of a single particle, and the second described the breakage of a particulate population using statistical methods. The investigation utilised quasi-static compression testing of individual particles, and trials in a pneumatic conveying rig (Figure 14). Superficial air velocities up to $20 \mathrm{~m} / \mathrm{s}$ were considered along with a range of conveying pipeline geometries. Particle fatigue and inter-particle collisions were not accounted for as sources of particle damage. 


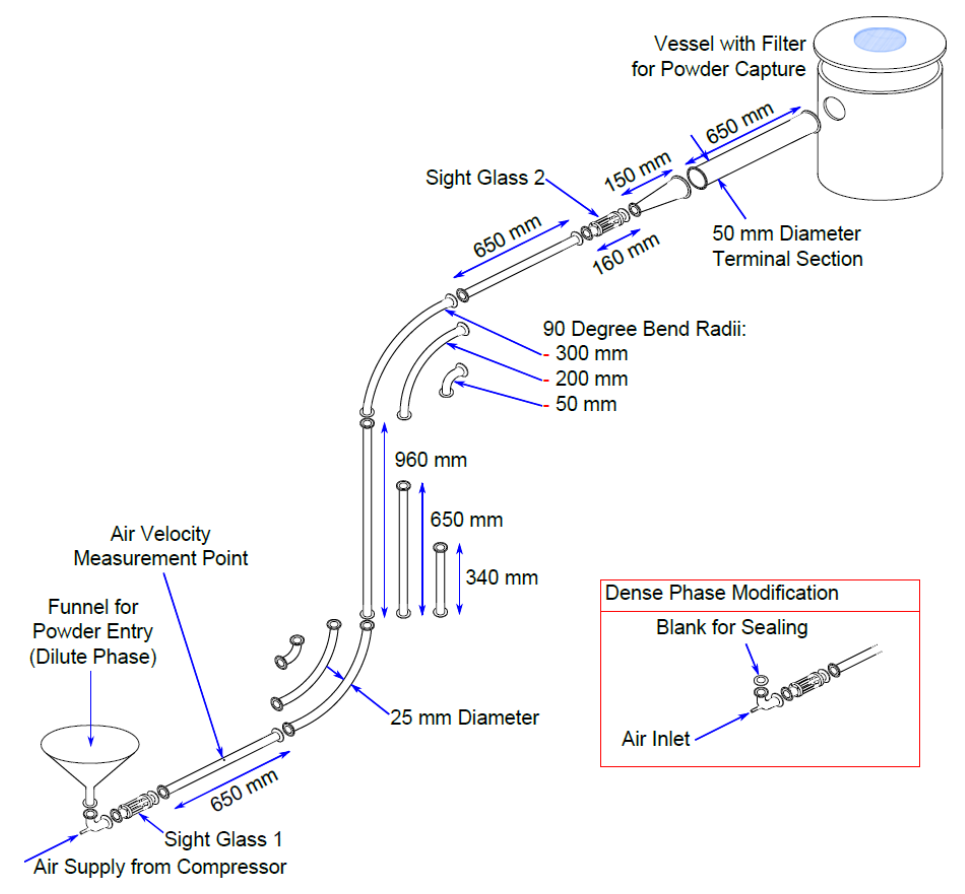

Figure 14: Pneumatic Conveying Rig used by Hanley [158]

Investigation of the variation in bulk density of infant formula through pneumatic conveying was presented by Hanley et al. [6]. This variation of bulk density was attributed to the breakage of particles through such processes. The Taguchi Method of experimental design and ANOVA analysis of the experimental results were utilised, in addition to the generation of polymeric models and subsequent analysis. It was concluded that the mode of conveying (dense/lean phase), air velocity and number of passes (particle fatigue), were all statistically significant factors with respect to variation of the bulk density of the material.

Particle impact at pipeline bends within lean phase pneumatic conveying systems was described stochastically by Hanley et al. [94]. Through the use of a Weibull distribution to describe the initial particle strength distribution, a uniform particle distribution at the pipe inlet, and assuming a single impact within a pneumatic conveying bend, the breakage probability of agglomerate particles was reported. The fluid flow was described by a model presented in a companion paper [159]. These results were compared with Monte Carlo Simulations, and the mean of the impact angle compared well, although a discrepancy of $20 \%$ was reported for the standard deviations.

Brosh et al. [160-162]

Brosh et al. [160] addressed the challenges relating to spawning child particles in the scenario where a mother particle is fractured. These challenges were specified as the allocation of initial conditions for each of the new child particles, and the subsequent interaction of these particles. The model developed in this paper was able to accept the use of any breakage function, and unlimited specification of resulting fragment size fractions. The model was applied to a jet milling process with reasonable correlation.

The above model was then applied [161] in the context of pneumatic conveying. The overall method of predicting particle breakage used in this simulation was composed of four sub-functions: initial strength distribution, equivalence/selection, fatigue, and breakage functions. Combined with a 3D CFD-DEM model, particle attrition was predicted for potash within a pneumatic conveying line 
containing four blind-T bends. The results were compared to experimental data, and it was found that the model over-predicted the degree of attrition occurring in the pipeline. Subsequently, methods for accelerating such simulations are presented [162], and include approaches such as the construction of a DEM grid, in which the empty cells were disregarded. Additionally, a sub-DEM grid was constructed for comminution calculations and with the same approach, the calculation could be further accelerated. Stiffness reduction of the smallest particles (or total removal of the smallest particles from the simulation) was also found to improve calculation speed, as the time step could be increased.

Uzi et al. [33]

Uzi et al. [33] presented a model of particle attrition within pneumatic conveying systems that adopted the two-phase flow model of Levy et al. [163], and applied a new one-dimensional breakage algorithm to describe the breakage behaviour. A known particle size distribution at the inlet of the pipeline was used, and by selecting a 'tracking mass', progressively calculated the changes in the size distribution along the pipe length. The pipeline was divided into elements, and a collision detection algorithm was used in each element. Impact conditions were compared with the strength of the particle (obtained from empirical analysis). If the particle strength was insufficient to withstand the collision, child particles were generated, however, if the particle was able to withstand the collision, a fatigue algorithm was employed for particle strength reduction. A block diagram describing the model is shown in Figure 15. Validation of the model was performed through comparison with the results obtained by Kalman et al. [127] and an absolute deviation was found to be $5.8 \%$ on average. Furthermore, particle-particle interactions were found to have significant influence on the results, especially in the bends of the pipeline.

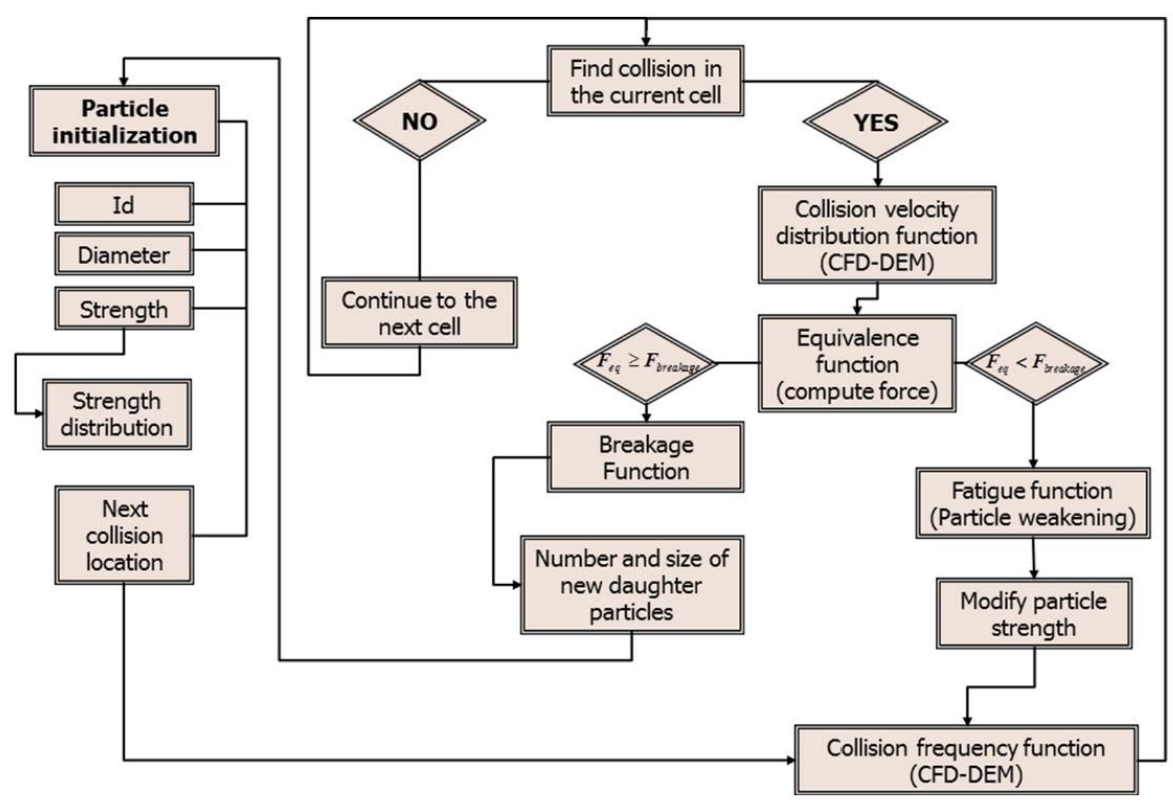

Figure 15: Block Diagram of the One-Dimensional Breakage Algorithm as described by Uzi et al. [33] 


\subsection{Empirical Approaches}

Empirical approaches to modelling particle attrition within lean phase pneumatic conveying systems are considered to be those which employ a data-fitting model. Presented here are a selection of key studies, organised by research group and year.

\section{Salman [104]}

Salman et al. [104] proposed a model for predicting the particle breakage characteristics during leanphase pneumatic conveying, combining a particle trajectory model [164] with empirically-derived breakage relationships. This study focused on single-particle impacts across a range of particle sizes, impact angle, mean transportation velocities, and fatigue behaviour. Two testers were used to obtain the experimental results; the first tester was similar to that of a gas blast tester, and the second consisted of a pneumatic conveying line with a single bend. Breakage data was collected in the 'gas blast' tester through manual examination of the tested particles under known conditions, and each of the data points was represented by the average of 100 test particles. Particles were classed as 'broken' or 'unbroken'. A two-parameter Weibull distribution was fitted to the test data as shown in Equation (4), where $N_{o}$ is the number of unbroken particles, $C_{p}$ is the particle impact velocity, and $c$ and $m$ are curve fit parameters.

$$
N_{o}=100 e^{-\left(\frac{C_{p}}{c}\right)^{m}}
$$

The conclusions are limited to particles of a near-spherical geometry The necessary acceleration length as reported by other researchers varies from $4-5 m$ to $6.5-9.3$ [20,51], and the longest acceleration length in this research is $4.5 \mathrm{~m}$. This implies that there is scope for further validation with industrial pneumatic conveying systems.

\section{Aked et al. [165], Kalman and Goder [166], and Kalman [4,5]}

Aked et al. [165] looked at the conveying behaviours of five different pneumatic conveying bend types with respect to pressure drop, particle attrition, and material build-up on the pipe walls. The study concluded that the observed particle attrition increased with increasing superficial air velocity, and decreased with increasing solids mass flow rate. These findings support the concept that particle 'shielding occurs at higher particle concentrations.

Kalman and Goder [166] applied the failure design theory for machine parts, to particle attrition behaviour. Single and multi-particle tests were considered across impact, compression and fatigue loading conditions. Experimentation was conducted through a 0.7 inch, $6 \mathrm{~m}$ pneumatic conveying line that contained 6 bends. General comments are made with respect to superficial air velocity, and bend type in line with reasonable expectations (i.e. increased particle attrition with increased number of loading cycles and increased superficial air velocity). A long radius flexible bend was found to inflict the lowest attrition rates on the potassium sulphate used in this work.

Kalman [4] considers the attrition mechanism within the context of controlling attrition levels through pneumatic conveying systems. It was concluded that by applying vibrations to a pneumatic conveying line with turbulence drums for directional changes, the measured degree of particle attrition could be reduced. Kalman [5] subsequently considers four different pneumatic conveying arrangements with various pipeline and bend geometries. It was demonstrated that the screw feeder used in two of the four testers generated more attrition than the pneumatic conveying line 
for particles larger than $3000 \mu \mathrm{m}$ in diameter. For particles smaller than $1000 \mu \mathrm{m}$ in diameter, it was found that less than $5 \%$ of the total attrition caused by the pneumatic conveying system could be attributed to the screw feeder. Such results indicate that particle size is of key importance when determining the level of article breakage attributed to a screw feeder, and subsequently, pneumatic conveying systems incorporating these feeders. Attrition caused by a cyclone separator was found to be up to $20 \%$ of the total attrition occurring within the system. Particle attrition was found to decrease with increased solids loading ratio. Whilst only qualitative observation is presented, results are in support of previous works, and the diverse range of materials tested demonstrate the variable nature of particle attrition within such systems.

Bridle [22]

Bridle [22] presents a study specific to particle attrition in lean phase pneumatic conveying. A bespoke single bend pneumatic conveying test line was designed and constructed (Figure 16), and the modification of an existing full-scale pneumatic conveying line was carried out. Three particulate materials were tested: sugar, malted barley, and basmati rice. The conveying conditions investigated in the single bend apparatus, along with their values are summarised in Table 2.

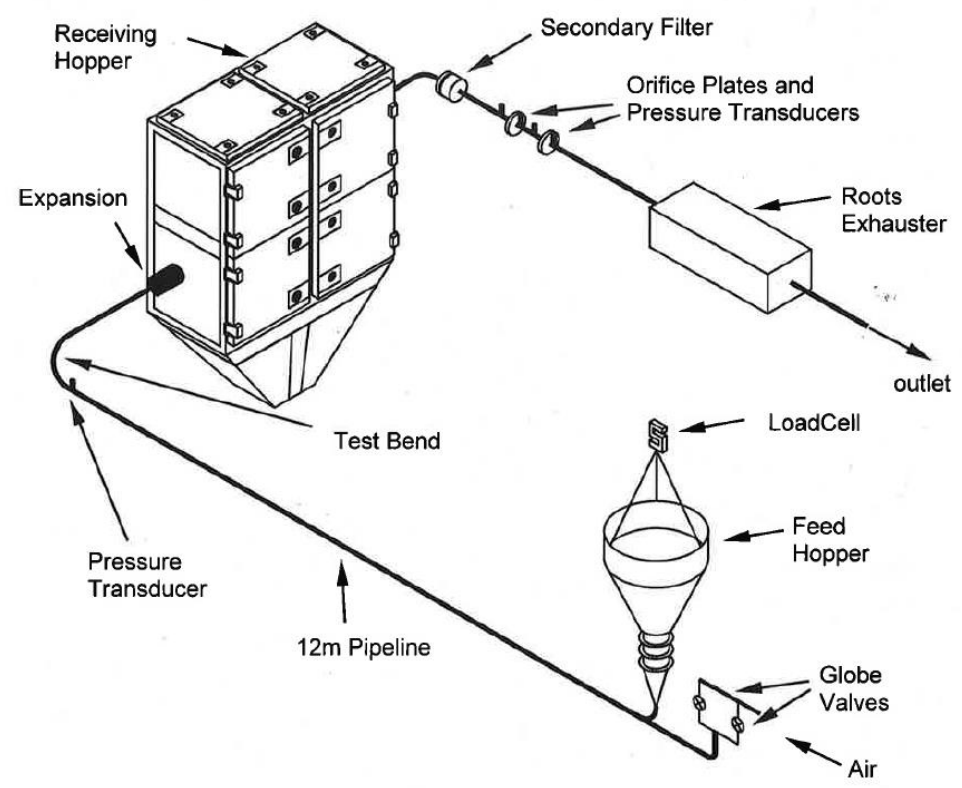

Figure 16: The Single Bend Test Apparatus as used by Bridle [22]

Table 2: Variables and associated values tested by Bridle [22] in the Single Bend Pneumatic Conveyor

\begin{tabular}{|l|l|}
\hline \multicolumn{1}{|c|}{ Conveying Variable } & \multicolumn{1}{c|}{ Values Tested } \\
\hline Bend geometry $(\mathrm{R} / \mathrm{d})$ & $0(B$ lind $\mathrm{T}), 3.6,13$ \\
\hline Superficial air velocity $(\mathrm{m} / \mathrm{s})$ & $9,16,22,26,30,34$, and 38 \\
\hline Suspension density $\left(\mathrm{kg} / \mathrm{m}^{3}\right)$ & $5,12,23,35$, and 60 \\
\hline
\end{tabular}

In order to simulate the conveying conditions in the full scale pneumatic conveying pipeline, the single bend test apparatus was used to replicate these conditions using only the short radius $(R / d=$ 3.6) bends. The amount of degradation observed in the single bend test facility was approximately double that measured in the full scale conveying system. The measurement criteria used for the comparison was the percentage fines generated below $300 \mu \mathrm{m}$ for sugar, percentage fines 
generated below $1200 \mu \mathrm{m}$ for barley and percentage of broken grains for the rice. A methodology is presented where a linear relationship between the magnitude of degradation observed in the single bend apparatus and the magnitude of degradation in the full scale conveying line is empirically calibrated. This method was demonstrated to give differences of up to $40 \%$, with data variances of up to $20 \%$. This work also forms the foundation of Bridle et al. [167].

\section{Frye and Peukert $[9,168-170]$}

This work was the subject of four publications, and focused on the study of the attrition behaviour of various polymers within lean phase pneumatic conveying systems. Two functions were used throughout the course of the research in order to define the attributes responsible for particle attrition: a process function, and a material function. The process function describes the loading conditions to which the particles are subjected, and the material function describes the reactive behaviour of the particles to these loading conditions.

Frye and Peukert $[168,169]$ use an Euler-Lagrange approach to predict the loading conditions that the particles are expected to be subjected to. The material function was then evaluated through conducting tests with a centrifugal impact tester and a modified pin-on-disc tribometer, resolving the material response to impact and sliding friction respectively. For the polymers under inspection, sliding friction was deemed to play a more significant role in the attrition of these particles than what has been previously reported in literature.

Subsequently, Frye and Peukert [170] numerically derived stress conditions and used them in conjunction with data from both of the previously mentioned test apparatuses. The results were compared with results obtained from a single test bend pneumatic conveying line apparatus. A significant variety of polymeric materials were tested in this work. Dynamic mechanical analysis (DMA), a technique whereby a pre-prepared sample of the material is cantilevered with a clamp and sinusoidally oscillated, was applied to each test material. It was concluded that the glass transition temperature of the material was of critical importance.

The final piece of research [9] that forms this series of work, follows on from the previous publications, and finds that the attrition of different polymeric materials is more closely resembled by different attrition mechanisms.

Abou-Chakra et al. [171] and Chapelle et al. [38,136,172]

Abou-Chakra et al. [171] applied the particle dynamics theory presented by Deng et al. [30] to a bench scale centrifugal attrition tester. Two crystalline materials were tested; using $10 \mathrm{~g}$ samples with two repetitions at each test condition. Experimental errors were reported to be below $3 \%$. As the impact velocity and impact angle were increased, the level of attrition was also observed to increase. To validate these results, the apparatus of Bridle [22] was used to determine the level of attrition caused by a single bend in a pneumatic conveying line, in addition to a full scale pneumatic conveying system. Good correlation was found at high impact velocities/conveying speeds.

This centrifugal attrition tester then formed the basis of another piece of work by Chapelle et al. [136], which presents a degradation model for particles subjected to lean-phase pneumatic conveying. This model utilises a breakage matrix approach drawing from population balance theory and is given in Equation (5). Where $[B]$ is the empirically-obtained breakage matrix, $\{i\}$ is the input particle size distribution going into the bend, and $\{o\}$ is the output particle size distribution going out of the bend. The latter two of these terms are defined by column matrices. An interpolation 
procedure is also presented for intermediate test conditions. Only impact angles of $90^{\circ}$ to the surface of the target were considered.

$$
[B] \cdot\{i\}=\{o\}
$$

Validation was presented in [172], where the degradation model was coupled with a two-phase flow model. The flow model specifies two regimes: strand flow, and fully-suspended flow. The model used for the strand flow was based on that presented by Bradley et al. [21], and the calculation progresses numerically along the pipeline which was divided into 'elements'. The concept of a 'harmonic mean size' of a particle size distribution was presented, as defined by Equation (6); where $X_{h}$ is the harmonic mean particle size, $w_{i}$ is the fraction of material retained on a given sieve size, and $d_{i}$ is the mean size of the size fraction correlating to $w_{i}$. The results of this model were compared against experimental results from both a single-bend pneumatic conveying laboratory test rig, and an industrial lean-phase pneumatic conveying pipeline. Good agreement was obtained between predicted and experimental results. Two different particle concentrations and air inlet velocities were considered.

$$
\frac{1}{X_{h}}=\sum \frac{w_{i}}{d_{i}}
$$

The final paper publication from Chapelle et al. [38] applies this degradation and flow model to two more industrial case studies. Both find good agreement with the experimental results.

Laarhoven et al. [106]

Through continued development of a new particle attrition tester (Figure 17) originally constructed by Beekman [173], and subsequent validation with a lean phase pneumatic conveying system, Laarhoven et al. [106] were able to correlate breakage patterns between the two loading methods for two types of fertiliser granule. A significant discrepancy between the two loading methods was that of impact velocity, which was accounted for by increasing the number of loading events. The mass specific kinetic energy was comparable across both systems for sodium benzoate granules where surface attrition dominated, up to a threshold of approximately $7 \mathrm{~kJ} / \mathrm{kg}$. Above this value, the results diverged. For scenarios where attrition occurred by particle fragmentation, no such correlation could be achieved. 


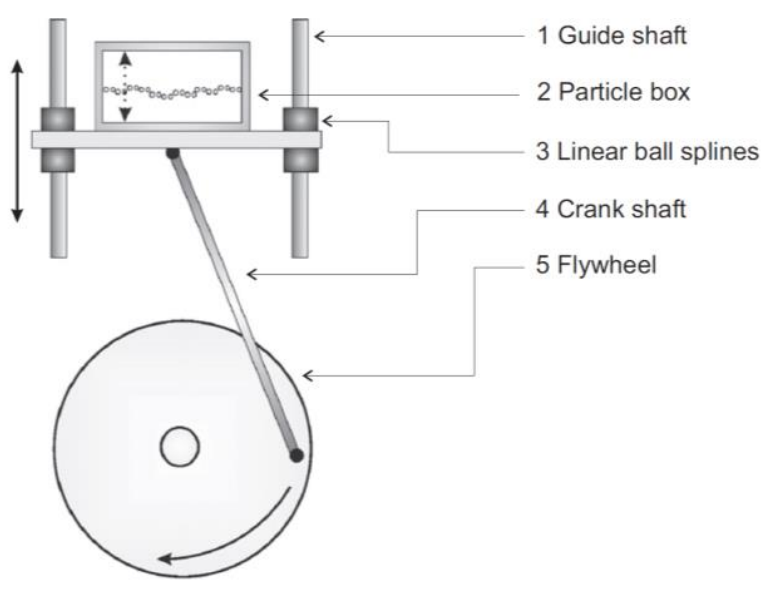

Figure 17: Particle Attrition Tester as used by Laarhoven et al.[106]

\section{Macchini et al. [36]}

Macchini et al. [36] considered the effect of particle size, density and concentration on the erosive wear caused to pipe bends in lean phase pneumatic conveying systems. While the particle attrition characteristics were not specifically investigated, as previously mentioned, pipe erosion and particle attrition are considered to be closely related as they are both results of the same impact event. The series of experiments used a full scale pneumatic conveying line with an insert in the test bend of interest (Figure 18), which could be removed for analysis. The key findings from this study were that the particle density was found to have a greater influence on the particle shielding effect in comparison to the particle size. When compared to the results from a centrifugal erosion tester, the erosion observed in the pneumatic conveying line was found to be smaller. Therefore a greater shielding effect was concluded to exist in the conveying line.

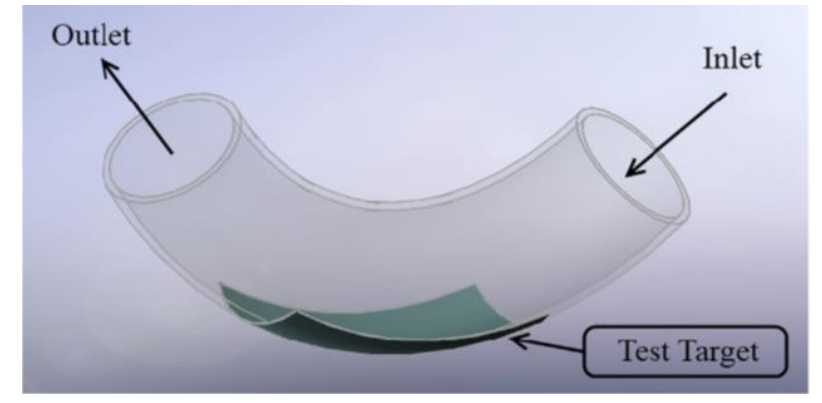

Figure 18: Test Bend used by Macchini et al. [36]

\subsection{Concluding Remarks on Modelling Approaches}

Through review of previous studies into particle attrition within lean phase pneumatic conveying systems, based on both empirical and theoretical approaches, it remains evident that considerable work is still required to understand the fundamental mechanisms involved. This observation is even more applicable when looking at the influence of particle concentration within the pipeline on particle attrition behaviour. Whilst in most studies the solids feed rate is specified, further research into how this interacts with the particulate material type and variation in pipeline geometry is required. It is evident from the review of these studies that factors such as superficial air velocity are 
well understood, however there is significant scope for further investigation with respect to particle concentration. Furthermore, particle velocity is not always measured. Considering the conclusions from Section 5.4.1.1, particle velocity is the most important factor in characterising particle attrition from the system perspective (as the impact angle is specified by system geometry). 


\section{Experimental Considerations}

This section will address experimental methodology specific to the sample preparation and size analysis of particulate materials.

\subsection{Sampling}

Sampling theory is of fundamental importance in the mass reduction and analysis of bulk materials, not least of all due to the fact that sampling errors typically far outweighing analytical errors [174176]. A quantitative approach was detailed by Gy [177-179], whereby extraction of correct samples was specified.

From a bulk solids perspective, there are a number of different mechanical subdividing machines available. Petersen et al. [180] conducted a critical survey of these devices. It was concluded that the best overall methods of mass reduction for particulate materials were the calibrated Boener divider, Rationel Kornservice A/S splitters containing 34 chutes, Rotating 32-divider, and a Vario Divider with a splitting ratio of $1: 4+1: 5$. These methods have been contrasted with the commonly used "grab sampling", which was concluded to be the worst for of mass reduction in terms of statistical representativeness.

One aspect of the Theory of Sampling to be aware of in attrition studies, is that particle size is inherent to the fundamental process under scrutiny. Therefore, Sampling Unit Operation (SUO) 5 as detailed by Petersen et al. [175], 'Comminution whenever necessary', cannot be applied to particle attrition research. Subsequently, alternative SUOs require application where feasible.

\subsection{Particle Size Measurement}

In many works, the method by which particle size is measured, is described in little or no detail. Such a key element in attrition studies requires considerable attention.

Kulkarni [181] presents an extensive review of particle size measurement by various sieving methods. There was insufficient or non-existent literature published on the influence of sieve diameter or amplitude of applied vibrations on the results obtained. Criticality was attributed to the sieving duration and sieving load. It was recommended to use a mass based system in data representation, without the use of size distribution modelling curves to smooth a measured size distribution. Presentation of the data in the form of histograms (showing the mass retained on each sieve) was deemed to provide more detailed size information, however the cumulative size distribution curve was deemed to better quantify the information holistically.

Standards describing the method by which solid particle size is to be measured with sieves are BS 410 (1986) (withdrawn), ISO 330-1 and ISO 3310-2. BS 410 was considered to be a pertinent standard [182], however BS 410-2:2000 remains in circulation, and outlines the technical requirements of testing and sieve geometry.

When considering other size measurement techniques such as Image Analysis, and Laser Diffraction, care must be taken to ensure that the technique is optimised for the particulate material under consideration. Li et al. [183] found that particle shape can significantly influence the measured particle size distribution output, depending on the method used. While spherical particle returned comparable results across three of the four measurement methods, non-spherical particle size distribution outputs could not be correlated (without the use of correlation factors obtained through image analysis). However, even for the case of spherical glass beads, differences between image analysis and laser diffraction have been reported by Bowen [184] as shown in Figure 19. 


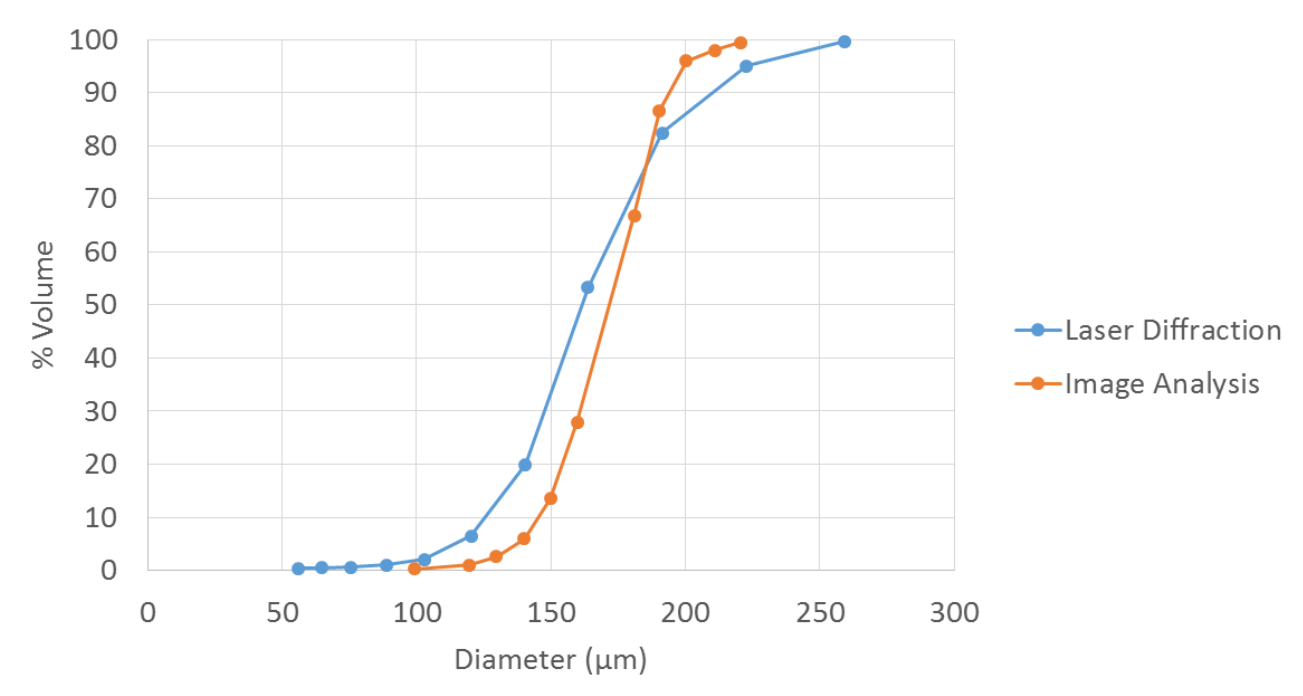

Figure 19: Comparison of laser diffraction and image analysis particle size distributions for sieved glass beads adapted from Bowen [184]

Not only the method by which particle size is measured is important, but also how that information is selected for presentation and how it is used. Some studies have used the cumulative particle size distribution curve previously mentioned, whilst other studies have adopted the use of the damage ratio (for example [185]). This was defined as the fraction of particle that are broken after being subjected to a defined loading condition, or number of loading conditions. Used predominantly in the modelling of comminution mechanisms, this was a simple measurement which must be defined for each particle type (size, shape, etc.). Such a function has been used to determine the probability of a given particle to break under a specified set of conditions, however, this does not produce the particle size distribution of the child particles. Further functions are required in this sense to describe the subsequent particle size distribution, similar to the breakage matrices presented by Chapelle et al. [38]. 


\section{The Future of the Field of Research}

In light of the culminated research presented above, there are some distinct areas of knowledge which contain gaps in understanding.

To further broaden the scope of the field, the method of production of the particulate material is rarely acknowledged. Only one paper specifically addresses the influence of the production process on the attrition behaviour of the particulate material [75]. Whilst manipulation of the process delves into the realms of formulation and chemical engineering, this is a vital part of understanding how the history of a particle dictates its mechanical properties and subsequent attrition behaviour.

Two distinct types of experimental investigation are evident in the literature: single particle analysis, and bulk behaviour analysis. Correlation between these two research areas is rarely reported in a quantitative form. Particle properties are always reported, but firm links between these properties and bulk behaviour are scarce. Pragmatically, these links offer the most realistic opportunity to apply research in the field, as plant operators seek cost effective design and troubleshooting solutions. To characterise a small particulate sample and subsequently predict bulk behaviour is of immense value (as the Jenike Shear Test has demonstrated for wall and internal friction [186]).

Each of the studies acknowledged in this review use different pieces of test equipment to obtain data. Whilst there are various advantages and disadvantages associated with each apparatus, this means that the results are specific to the study and cannot be directly compared with the results of other studies. Considering the vast array of materials and properties, there is a requirement for benchmarking material attrition behaviour in response to a standard set of test conditions.

Given that many different particle attrition test apparatus exist, very few studies have compared the data obtained from the different types (for example [187]). This analysis extends to resolving the forces applied to the particle and validating such an analysis through direct measurement. One example of such work is authored by Burnett et al. [132]. Ultimately, it is of great interest to develop a material characterisation method, which, when coupled with an appropriate model, makes possible the prediction of particle attrition within lean phase pneumatic conveying systems of any geometry. 


\section{Conclusions of the Review}

The phenomenon of particle attrition within lean phase pneumatic conveying was broken down categorically and has been systematically addressed. The primary categories addressed were the contributions of the material and process factors, and selected studies conducted from empirical and fundamentally derived approaches. Experimental considerations were also highlighted as an important factor in obtaining representative data. Throughout the course of this review, some key findings became evident:

1. Wear of wall surfaces and particle attrition are intimately connected. Both mechanisms result from the same contact event.

2. Particulate materials are diverse in nature, and fail by drastically different mechanisms dependant on their composition. Even particles residing within the same sample have been demonstrated to show a strength distribution. Furthermore, the failure mechanism was specific to the nature and magnitude of the loading event.

3. There remain a significant number of gaps in the research to be explored, especially with respect to linking the method of characterisation to the process under consideration.

4. The diversity of test equipment was highlighted, and concern was attributed to the lack of correlation between different equipment types.

5. The particle velocity is not measured in a vast number of pneumatic conveying research works when considering particle attrition. The velocity of the conveying air, while much easier to measure, should not be used to approximate particle velocity without understanding the slip velocity characteristics specific to the system and material. As particle velocity is repeatedly reported to be the most influential factor in particle attrition, it should be measured directly.

6. A successful particle attrition modelling approach will need to capture both the material and process functions of the phenomenon in order to achieve meaningful results.

In conclusion, understanding particle attrition mechanisms is of great value in their manifestation in processes such as lean phase pneumatic conveying. There are a significant number of avenues yet to be explored fully, however with the synthesis of information contained with this review, it is hoped that future research may be coherent and informed. 
[1] C.R. Bemrose, J. Bridgwater, A review of attrition and attrition test methods, Powder Technol. 49 (1987) 97-126. doi:10.1016/0032-5910(87)80054-2.

[2] I.M. Hutchings, Mechanisms of wear in powder technology: A review, Powder Technol. 76 (1993) 3-13. doi:10.1016/0032-5910(93)80035-9.

[3] G.K. Reynolds, J.S. Fu, Y.S. Cheong, M.J. Hounslow, A.D. Salman, Breakage in granulation: A review, Chem. Eng. Sci. 60 (2005) 3969-3992. doi:10.1016/j.ces.2005.02.029.

[4] H. Kalman, Attrition control by pneumatic conveying, Powder Technol. 104 (1999) 214-220. doi:10.1016/S0032-5910(99)00097-2.

[5] H. Kalman, Attrition of powders and granules at various bends during pneumatic conveying, Powder Technol. 112 (2000) 244-250. doi:10.1016/S0032-5910(00)00298-9.

[6] K.J. Hanley, E.P. Byrne, K. Cronin, J.C. Oliveira, J. a. O'Mahony, M. a. Fenelon, Effect of pneumatic conveying parameters on physical quality characteristics of infant formula, J. Food Eng. 106 (2011) 236-244. doi:10.1016/j.jfoodeng.2011.04.029.

[7] H. Akilli, E.. Levy, B. Sahin, Gas-solid flow behavior in a horizontal pipe after a $90^{\circ}$ vertical-tohorizontal elbow, Powder Technol. 116 (2001) 43-52. doi:10.1016/S0032-5910(00)00360-0.

[8] W. Yang, B. Kuan, Experimental investigation of dilute turbulent particulate flow inside a curved bend, Chem. Eng. Sci. 61 (2006) 3593-3601. doi:10.1016/j.ces.2006.01.013.

[9] L. Frye, W. Peukert, Identification of material specific attrition mechanisms for polymers in dilute phase pneumatic conveying, Chem. Eng. Process. Process Intensif. 44 (2005) 175-185. doi:10.1016/j.cep.2004.03.012.

[10] H. Li, Y. Tomita, Particle velocity and concentration characteristics in a horizontal dilute swirling flow pneumatic conveying, Powder Technol. 107 (2000) 144-152. doi:10.1016/S0032-5910(99)00181-3.

[11] A. Rinoshika, F. Yan, M. Kikuchi, Experimental study on particle fluctuation velocity of a horizontal pneumatic conveying near the minimum conveying velocity, Int. J. Multiph. Flow. 40 (2012) 126-135. doi:10.1016/j.ijmultiphaseflow.2011.11.007.

[12] D.G. Papadopoulos, M. Ghadiri, Impact breakage of poly-methylmethacrylate (PMMA) extrudates: I. Chipping mechanism, Adv. Powder Technol. 7 (1996) 183-197. doi:10.1016/S0921-8831(08)60495-1.

[13] K.R. Yuregir, M. Ghadiri, R. Clift, Observations on impact attrition of granular solids, Powder Technol. 49 (1986) 53-57. doi:10.1016/0032-5910(86)85004-5.

[14] A.. Salman, J. Fu, D.. Gorham, M.. Hounslow, Impact breakage of fertiliser granules, Powder Technol. 130 (2003) 359-366. doi:10.1016/S0032-5910(02)00237-1.

[15] C. Xu, J. Li, S. Wang, A spatial filtering velocimeter for solid particle velocity measurement based on linear electrostatic sensor array, Flow Meas. Instrum. 26 (2012) 68-78. doi:10.1016/j.flowmeasinst.2012.03.006.

[16] J. Li, C. Xu, S. Wang, Spatial filtering characteristics of electrostatic sensor matrix for local velocity measurement of pneumatically conveyed particles, Measurement. 53 (2014) 194205. doi:10.1016/j.measurement.2014.03.037.

[17] N. Huber, M. Sommerfeld, Modelling and numerical calculation of dilute-phase pneumatic conveying in pipe systems, Powder Technol. 99 (1998) 90-101. doi:10.1016/S00325910(98)00065-5. 
[18] W. Wei, G. Qingliang, W. Yuxin, Y. Hairui, Z. Jiansheng, L. Junfu, Experimental study on the solid velocity in horizontal dilute phase pneumatic conveying of fine powders, Powder Technol. 212 (2011) 403-409. doi:10.1016/j.powtec.2011.06.014.

[19] I.. Barratt, Y. Yan, B. Byrne, M.S.. Bradley, Mass flow measurement of pneumatically conveyed solids using radiometric sensors, Flow Meas. Instrum. 11 (2000) 223-235. doi:10.1016/S0955-5986(00)00022-4.

[20] P.J. Mendies, J.N. Wheeldon, J.C. Williams, The Velocity of Granular Material Flowing in a Pneumatic Conveyor, in: Proc. Pneumotransp. 2 Second Int. Conf. Pneum. Transp. Solids Pipes, 1973.

[21] M.S.A. Bradley, R.J. Farnish, L.M. Hyder, A.R. Reed, Handbook of Conveying and Handling of Particulate Solids, Elsevier, 2001. doi:10.1016/S0167-3785(01)80043-9.

[22] I. Bridle, The Analysis of Particle Degradation in Pneumatic Conveyors Utilizing a Pilot-Sized Test Facility, University of Greenwich, 2000.

[23] M.S.A. Bradley, Prediction of Pressure Losses in Pneumatic Conveying Pipelines, Thames Polytechnic, 1990.

[24] P.W. Wypych, P.C. Arnold, On improving scale-up procedures for pneumatic conveying design, Powder Technol. 50 (1987) 281-294. doi:10.1016/0032-5910(87)80074-8.

[25] P.W. Wypych, Pneumatic Conveying of Bulk Solids, University of Wollongong, 1989.

[26] Z.F. Tian, K. Inthavong, J.Y. Tu, G.H. Yeoh, Numerical investigation into the effects of wall roughness on a gas-particle flow in a $90^{\circ}$ bend, Int. J. Heat Mass Transf. 51 (2008) 12381250. doi:10.1016/j.ijheatmasstransfer.2007.12.005.

[27] M. Sommerfeld, J. Kussin, Wall roughness effects on pneumatic conveying of spherical particles in a narrow horizontal channel, Powder Technol. 142 (2004) 180-192. doi:10.1016/j.powtec.2004.05.002.

[28] K.A. Aarseth, Attrition of Feed Pellets during Pneumatic Conveying: the Influence of Velocity and Bend Radius, Biosyst. Eng. 89 (2004) 197-213. doi:10.1016/j.biosystemseng.2004.06.008.

[29] N. Zumaeta, E.P. Byrne, J.J. Fitzpatrick, Predicting precipitate breakage during turbulent flow through different flow geometries, Colloids Surfaces A Physicochem. Eng. Asp. 292 (2007) 251-263. doi:10.1016/j.colsurfa.2006.06.032.

[30] T. Deng, M.S.. Bradley, M.. Bingley, An investigation of particle dynamics within a centrifugal accelerator type erosion tester, Wear. 247 (2001) 55-65. doi:10.1016/S0043-1648(00)005147.

[31] Y. Zheng, Q. Liu, Review of techniques for the mass flow rate measurement of pneumatically conveyed solids, Meas. J. Int. Meas. Confed. 44 (2011) 589-604. doi:10.1016/j.measurement.2011.01.013.

[32] Y. Yan, Mass flow measurement of bulk solids in pneumatic pipelines, Meas. Sci. Technol. 7 (1996) 1687-1706. doi:10.1088/0957-0233/7/12/002.

[33] A. Uzi, H. Kalman, A. Levy, A novel particle attrition model for conveying systems, Powder Technol. 298 (2016) 30-41. doi:10.1016/j.powtec.2016.05.014.

[34] B. Kuan, W. Yang, M.P. Schwarz, Dilute gas-solid two-phase flows in a curved duct bend: CFD simulation with experimental validation, Chem. Eng. Sci. 62 (2007) 2068-2088. doi:10.1016/j.ces.2006.12.054. 
[35] D. Mills, M.G. Jones, V.K. Agarwal, Handbook of Pneumatic Conveying Engineering, 2004.

[36] R. Macchini, M.S. a. Bradley, T. Deng, Influence of particle size, density, particle concentration on bend erosive wear in pneumatic conveyors, Wear. 303 (2013) 21-29. doi:10.1016/j.wear.2013.02.014.

[37] J. Ma, Y. Yan, Design and evaluation of electrostatic sensors for the measurement of velocity of pneumatically conveyed solids, Flow Meas. Instrum. 11 (2000) 195-204. doi:10.1016/S0955-5986(00)00019-4.

[38] P. Chapelle, H. Abou-Chakra, N. Christakis, I. Bridle, M.K. Patel, J. Baxter, U. Tuzun, M. Cross, Numerical predictions of particle degradation in industrial-scale pneumatic conveyors, Powder Technol. 143-144 (2004) 321-330. doi:10.1016/j.powtec.2004.04.024.

[39] T. Deng, A.R. Chaudhry, M. Patel, I. Hutchings, M.S.A. Bradley, Effect of particle concentration on erosion rate of mild steel bends in a pneumatic conveyor, Wear. 258 (2005) 480-487. doi:10.1016/j.wear.2004.08.001.

[40] H.L. Hu, T.M. Xu, S.E. Hui, Q.L. Zhou, A novel capacitive system for the concentration measurement of pneumatically conveyed pulverized fuel at power stations, Flow Meas. Instrum. 17 (2006) 87-92. doi:10.1016/j.flowmeasinst.2005.11.001.

[41] Twin-array capacitance sensor for multi-parameter measurements of multiphase flow, Particuology. 22 (2015) 163-176. doi:10.1016/J.PARTIC.2014.06.014.

[42] J. Mennell, B. Byrne, Y. Yan, Appraisal of radiometric techniques to determine absolute solids fraction in pneumatic suspensions of particulate solids, Flow Meas. Instrum. 11 (2000) 213221. doi:10.1016/S0955-5986(00)00021-2.

[43] Y. Van, B. Byrne, J. Coulthard, Radiation attenuation of pulverised fuel in pneumatic conveying systems, Trans. Inst. Meas. Control. 15 (1993) 98-103. doi:10.1177/014233129301500301.

[44] J. Mennell, B. Byrne, Y. Yan, Non-invasive two phase flow measurement using soft X-ray attenuation, IEE Colloq. (1996) 9/1-9/3. https://www.scopus.com/inward/record.uri?eid=2s2.0-0030386764\&partnerID=40\&md5=858b7cb1fd3875b66b70a1223bb17a79.

[45] X. Chen, W. Zhou, X. Cai, M. Su, H. Liu, In-line imaging measurements of particle size, velocity and concentration in a particulate two-phase flow, Particuology. 13 (2014) 106-113. doi:10.1016/j.partic.2013.03.005.

[46] K. Miyazaki, G. Chen, F. Yamamoto, PIV measurement of particle motion in spiral gas \pm solid two-phase ${ }^{-}$ow, 19 (1999) 194-203.

[47] A.J. Burnett, The use of laboratory erosion tests for the prediction of wear in pneumatic conveying bends, University of Greenwich, 1996.

[48] S. Laín, M. Sommerfeld, Numerical calculation of pneumatic conveying in horizontal channels and pipes: Detailed analysis of conveying behaviour, Int. J. Multiph. Flow. 39 (2012) 105-120. doi:10.1016/j.ijmultiphaseflow.2011.09.006.

[49] S. Fokeer, S. Kingman, I. Lowndes, a Reynolds, Characterisation of the cross sectional particle concentration distribution in horizontal dilute flow conveying-a review, Chem. Eng. Process. Process Intensif. 43 (2004) 677-691. doi:10.1016/S0255-2701(03)00096-5.

[50] P. Middha, B. V. Balakin, L. Leirvaag, A.C. Hoffmann, P. Kosinski, PEPT - A novel tool for investigation of pneumatic conveying, Powder Technol. 237 (2013) 87-96. doi:10.1016/j.powtec.2013.01.024. 
[51] A.R. Chaudhry, Prediction of Bend Pressure Losses in Horizontal Lean Phase Pneumatic Conveying, University of Greenwich, 2004.

[52] Y. Ding, Z. Wang, D. Wen, M. Ghadiri, X. Fan, D. Parker, Solids behaviour in a dilute gas-solid two-phase mixture flowing through monolith channels, Chem. Eng. Sci. 61 (2006) 1561-1570. doi:10.1016/j.ces.2005.09.013.

[53] C.A.R. Duarte, F.J. de Souza, V.F. dos Santos, Mitigating elbow erosion with a vortex chamber, Powder Technol. 288 (2016) 6-25. doi:10.1016/j.powtec.2015.10.032.

[54] D.R. Andrews, N. Horsfield, Particle collisions in the vicinity of an eroding surface, J. Phys. D. Appl. Phys. 16 (1983) 525-538. doi:10.1088/0022-3727/16/4/014.

[55] M. Ghadiri, Z. Zhang, Impact attrition of particulate solids. Part 1: A theoretical model of chipping, Chem. Eng. Sci. 57 (2002) 3659-3669. doi:10.1016/S0009-2509(02)00240-3.

[56] E.N. Hiestand, C.B. Peot, Tensile Strength of Compressed Powders and an Example of Incompatibility as End-Point on Shear Yield Locus, J. Pharm. Sci. 63 (1974) 605-612. doi:10.1002/jps.2600630426.

[57] A.A. Griffith, The Phenomena of Rupture and Flow in Solids, Philos. Trans. R. Soc. London A Math. Phys. Eng. Sci. 221 (1921) 163-198.

http://rsta.royalsocietypublishing.org/content/221/582-593/163.abstract.

[58] B.W. Darvell, Uniaxial compression tests and the validity of indirect tensile strength, J. Mater. Sci. 25 (1990) 757-780.

https://www.engineeringvillage.com/share/document.url?mid=cpx_3777071\&database=cpx.

[59] H.M. Pollock, D. Maugis, M. Barquins, CHARACTERIZATION OF SUBMICROMETRE SURFACE LAYERS BY INDENTATION., ASTM Spec. Tech. Publ. (1985) 47-71.

https://www.engineeringvillage.com/share/document.url?mid=cpx_2217792\&database=cpx.

[60] D.G. Bika, M. Gentzler, J.N. Michaels, Mechanical properties of agglomerates, Powder Technol. 117 (2001) 98-112. doi:10.1016/S0032-5910(01)00318-7.

[61] S.M. Iveson, J.D. Litster, Liquid-bound granule impact deformation and coefficient of restitution, Powder Technol. 99 (1998) 234-242. doi:10.1016/S0032-5910(98)00115-6.

[62] J.B. Hawkyard, A theory for the mushrooming of flat-ended projectiles impinging on a flat rigid anvil, using energy considerations, Int. J. Mech. Sci. 11 (1969) 313-333. doi:10.1016/0020-7403(69)90049-6.

[63] E.E. Gdoutos, Fracture Mechanics: An Introduction, Book. 123 (2005) 369. http://books.google.com/books?id=MBMv6Pru4tgC\&hl=zh-CN.

[64] ASMT E399-90, Standard Test Method for Plane-Strain Fracture Toughness of Metallic Materials, ASTM Int. (1997) 1-31. doi:10.1520/E0399-09E02.2.

[65] J. Subero, M. Ghadiri, Breakage patterns of agglomerates, Powder Technol. 120 (2001) 232243. doi:10.1016/S0032-5910(01)00276-5.

[66] G.R. Irwin, Analysis of Stresses and Strains Near the End of a Crack Traversing a Plate, J. Appl. Mech. 24 (1957) 361-364. doi:no DOI.

[67] N. Ouchiyama, J.J. Benbow, J. Bridgwater, On the fracture toughness of extrudates and its relationship to rates of bulk particle attrition, Powder Technol. 51 (1987) 103-114. doi:10.1016/0032-5910(87)80043-8. 
[68] S. Antonyuk, J. Tomas, S. Heinrich, L. Mörl, Breakage behaviour of spherical granulates by compression, Chem. Eng. Sci. 60 (2005) 4031-4044. doi:10.1016/j.ces.2005.02.038.

[69] B.. Mishra, C. Thornton, Impact breakage of particle agglomerates, Int. J. Miner. Process. 61 (2001) 225-239. doi:10.1016/S0301-7516(00)00065-X.

[70] F.K. Wittel, H. a. Carmona, F. Kun, H.J. Herrmann, Mechanisms in impact fragmentation, Int. J. Fract. 154 (2009) 105-117. doi:10.1007/s10704-008-9267-6.

[71] Z.B. Tong, R.Y. Yang, A.B. Yu, S. Adi, H.K. Chan, Numerical modelling of the breakage of loose agglomerates of fine particles, Powder Technol. 196 (2009) 213-221.

doi:10.1016/j.powtec.2009.08.001.

[72] K.. Kafui, C. Thornton, Numerical simulations of impact breakage of a spherical crystalline agglomerate, Powder Technol. 109 (2000) 113-132. doi:10.1016/S0032-5910(99)00231-4.

[73] Z. Ning, R. Boerefijn, M. Ghadiri, C. Thornton, Distinct element simulation of impact breakage of lactose agglomerates, Adv. Powder Technol. 8 (1997) 15-37. doi:10.1016/S09218831(08)60477-X.

[74] A.. Salman, G.. Reynolds, J.. Fu, Y.. Cheong, C.. Biggs, M.. Adams, D.. Gorham, J. Lukenics, M.. Hounslow, Descriptive classification of the impact failure modes of spherical particles, Powder Technol. 143-144 (2004) 19-30. doi:10.1016/j.powtec.2004.04.005.

[75] M. Ghadiri, K.R. Yuregir, H.M. Pollock, J.D.J. Ross, N. Rolfe, Influence of processing conditions on attrition of $\mathrm{NaCl}$ crystals, Powder Technol. 65 (1991) 311-320. doi:10.1016/00325910(91)80195-O.

[76] J.A.S. Cleaver, M. Ghadiri, N. Rolfe, Impact attrition of sodium carbonate monohydrate crystals, Powder Technol. 76 (1993) 15-22. doi:10.1016/0032-5910(93)80036-A.

[77] M. Thomas, A.F.B. van der Poel, Physical quality of pelleted animal feed 1 . Criteria for pellet quality, Anim. Feed Sci. Technol. 61 (1996) 89-112. doi:10.1016/0377-8401(96)00949-2.

[78] M. Thomas, D.J. van Zuilichem, A.F.B. van der Poel, Physical quality of pelleted animal feed. 2. contribution of processes and its conditions, Anim. Feed Sci. Technol. 64 (1997) 173-192. doi:10.1016/S0377-8401(96)01058-9.

[79] M. Thomas, T. van Vliet, A.F.B. van der Poel, Physical quality of pelleted animal feed 3. Contribution of feedstuff components, Anim. Feed Sci. Technol. 70 (1998) 59-78. doi:10.1016/S0377-8401(97)00072-2.

[80] R. Seifried, W. Schiehlen, P. Eberhard, Numerical and experimental evaluation of the coefficient of restitution for repeated impacts, Int. J. Impact Eng. 32 (2005) 508-524. doi:10.1016/j.ijimpeng.2005.01.001.

[81] D.B. Hastie, Experimental measurement of the coefficient of restitution of irregular shaped particles impacting on horizontal surfaces, Chem. Eng. Sci. 101 (2013) 828-836. doi:10.1016/j.ces.2013.07.010.

[82] J. Fu, M.J. Adams, G.K. Reynolds, A.D. Salman, M.J. Hounslow, Impact deformation and rebound of wet granules, Powder Technol. 140 (2004) 248-257. doi:10.1016/j.powtec.2004.01.012.

[83] C. Mangwandi, Y.S. Cheong, M.J. Adams, M.J. Hounslow, A.D. Salman, The coefficient of restitution of different representative types of granules, Chem. Eng. Sci. 62 (2007) 437-450. doi:10.1016/j.ces.2006.08.063. 
[84] S.. Wu, K.. Chau, T.. Yu, Crushing and fragmentation of brittle spheres under double impact test, Powder Technol. 143-144 (2004) 41-55. doi:10.1016/j.powtec.2004.04.028.

[85] J. Fu, G.K. Reynolds, M.J. Adams, M.J. Hounslow, A.D. Salman, An experimental study of the impact breakage of wet granules, Chem. Eng. Sci. 60 (2005) 4005-4018. doi:10.1016/j.ces.2005.02.037.

[86] S.Z. Wu, K.T. Chau, Dynamic response of an elastic sphere under diametral impacts, Mech. Mater. 38 (2006) 1039-1060. doi:10.1016/j.mechmat.2005.08.005.

[87] L. Liu, K.D. Kafui, C. Thornton, Impact breakage of spherical, cuboidal and cylindrical agglomerates, Powder Technol. 199 (2010) 189-196. doi:10.1016/j.powtec.2010.01.007.

[88] Z. Zhang, M. Ghadiri, Impact attrition of particulate solids. Part 2: Experimental work, Chem. Eng. Sci. 57 (2002) 3671-3686. doi:10.1016/S0009-2509(02)00241-5.

[89] S. Antonyuk, S. Palis, S. Heinrich, Breakage behaviour of agglomerates and crystals by static loading and impact, Powder Technol. 206 (2011) 88-98. doi:10.1016/j.powtec.2010.02.025.

[90] S. Aman, J. Tomas, H. Kalman, Breakage probability of irregularly shaped particles, Chem. Eng. Sci. 65 (2010) 1503-1512. doi:10.1016/j.ces.2009.10.016.

[91] H. Briesen, Two-dimensional population balance modeling for shape dependent crystal attrition, Chem. Eng. Sci. 64 (2009) 661-672. doi:10.1016/j.ces.2008.05.002.

[92] L.G. Austin, The effect of damage on breakage kinetics, Powder Technol. 143-144 (2004) 151-159. doi:10.1016/j.powtec.2004.04.048.

[93] W. Weibull, A statistical theory of the strength of materials, R. Swedish Inst. Eng. Res. 151 (1939) 1-45.

[94] K.J. Hanley, E.P. Byrne, K. Cronin, Probabilistic analysis of particle impact at a pipe bend in pneumatic conveying, Powder Technol. 233 (2013) 176-185. doi:10.1016/j.powtec.2012.08.018.

[95] K.A. Aarseth, E. Prestløkken, Mechanical Properties of Feed Pellets: Weibull Analysis, Biosyst. Eng. 84 (2003) 349-361. doi:10.1016/S1537-5110(02)00264-7.

[96] A. Paluszny, X. Tang, M. Nejati, R.W. Zimmerman, A direct fragmentation method with Weibull function distribution of sizes based on finite- and discrete element simulations, Int. J. Solids Struct. 80 (2016) 38-51. doi:10.1016/j.ijsolstr.2015.10.019.

[97] W. Weibull, A statistical distribution function of wide applicability, J. Appl. Mech. 18 (1951) 293-297. doi:citeulike-article-id:8491543.

[98] A. Salman, D. Gorham, A. Verba, A study of solid particle failure under normal and oblique impact, Wear. 187 (1995) 92-98.

http://www.sciencedirect.com/science/article/pii/0043164895071407 (accessed October 23, 2015).

[99] A. Johansen, T. Schafer, Effects of interactions between powder particle size and binder viscosity on agglomerate growth mechanisms in a high shear mixer, Eur. J. Pharm. Sci. 12 (2000) 297-309. doi:10.1016/S0928-0987(00)00182-2.

[100] S.T. Keningley, P.C. Knight, A.D. Marson, An investigation into the effects of binder viscosity on agglomeration behaviour, Powder Technol. 91 (1997) 95-103. doi:10.1016/S00325910(96)03230-5. 
[101] K. Schönert, Breakage of spheres and circular discs, Powder Technol. 143-144 (2004) 2-18. doi:10.1016/j.powtec.2004.04.004.

[102] B.R. Lawn, M. V. Swain, Microfracture beneath point indentations in brittle solids, J. Mater. Sci. 10 (1975) 113-122. doi:10.1007/BF00541038.

[103] P.M.M. Vervoorn, L.G. Austin, The analysis of repeated breakage events as an equivalent rate process, Powder Technol. 63 (1990) 141-147. doi:10.1016/0032-5910(90)80036-X.

[104] A.D. Salman, M.J. Hounslow, A. Verba, Particle fragmentation in dilute phase pneumatic conveying, Powder Technol. 126 (2002) 109-115. doi:10.1016/S0032-5910(02)00048-7.

[105] Y.S. Cheong, A.D. Salman, M.J. Hounslow, Effect of impact angle and velocity on the fragment size distribution of glass spheres, Powder Technol. 138 (2003) 189-200. doi:10.1016/j.powtec.2003.09.010.

[106] B. van Laarhoven, S.H. Schaafsma, G.M.H. Meesters, Toward a desktop attrition tester; validation with dilute phase pneumatic conveying, Chem. Eng. Sci. 73 (2012) 321-328. doi:10.1016/j.ces.2011.08.038.

[107] A. Samimi, R. Moreno, M. Ghadiri, Analysis of impact damage of agglomerates: effect of impact angle, Powder Technol. 143-144 (2004) 97-109. doi:10.1016/j.powtec.2004.04.027.

[108] R. Moreno, M. Ghadiri, S.J. Antony, Effect of the impact angle on the breakage of agglomerates: a numerical study using DEM, Powder Technol. 130 (2003) 132-137. doi:10.1016/S0032-5910(02)00256-5.

[109] C. Subero-Couroyer, M. Ghadiri, N. Brunard, F. Kolenda, Weibull Analysis of Quasi-Static Crushing Strength of Catalyst Particles, Chem. Eng. Res. Des. 81 (2003) 953-962. doi:http://dx.doi.org/10.1205/026387603322482194.

[110] C. Couroyer, Attrition of Alumina Catalyst Carrier Beads, University of Surrey, 2000.

[111] K.T. Chau, X.X. Wei, R.H.C. Wong, T.X. Yu, Fragmentation of brittle spheres under static and dynamic compressions: experiments and analyses, Mech. Mater. 32 (2000) 543-554. doi:10.1016/S0167-6636(00)00026-0.

[112] S.M. Iveson, N.W. Page, J.D. Litster, The importance of wet-powder dynamic mechanical properties in understanding granulation, Powder Technol. 130 (2003) 97-101. doi:10.1016/S0032-5910(02)00233-4.

[113] D.. Gorham, A.. Salman, M.. Pitt, Static and dynamic failure of PMMA spheres, Powder Technol. 138 (2003) 229-238. doi:10.1016/j.powtec.2003.09.008.

[114] M. Ghadiri, Z. Ning, S.. Kenter, E. Puik, Attrition of granular solids in a shear cell, Chem. Eng. Sci. 55 (2000) 5445-5456. doi:10.1016/S0009-2509(00)00168-8.

[115] C.E.D. Ouwerkerk, A micro-mechanical connection between the single-particle strength and the bulk strength of random packings of spherical particles, Powder Technol. 65 (1991) 125138. doi:10.1016/0032-5910(91)80175-I.

[116] A.U. Neil, J. Bridgwater, Attrition of particulate solids under shear, Powder Technol. 80 (1994) 207-219. doi:10.1016/0032-5910(94)02849-4.

[117] C. Hare, M. Ghadiri, R. Dennehy, Prediction of attrition in agitated particle beds, Chem. Eng. Sci. 66 (2011) 4757-4770. doi:10.1016/j.ces.2011.06.042.

[118] A. D6175-03, Standard Test Method for Radial Crush Strength of Extruded Catalyst and 
Catalyst Carrier Particles, ASTM Int. (2013).

[119] M.. Chaudhri, Impact breakage of semi-brittle spheres, Powder Technol. 143-144 (2004) 3140. doi:10.1016/j.powtec.2004.04.006.

[120] P.H. Shipway, I.M. Hutchings, Attrition of brittle spheres by fracture under compression and impact loading, Powder Technol. 76 (1993) 23-30. doi:10.1016/0032-5910(93)80037-B.

[121] D. Goder, H. Kalman, a Ullmann, Fatigue characteristics of granular materials, Powder Technol. 122 (2002) 19-25. doi:10.1016/S0032-5910(01)00390-4.

[122] D. Goder, O. Eskin, H. Kalman, Fatigue Characteristics of Granular Materials, in: Third Isr. Conf. Conveying Handl. Part. Solids, 2000: p. 3.32-3.38.

[123] T. Han, H. Kalman, A. Levy, Theoretical and experimental study of multi-compression particle breakage, Adv. Powder Technol. 14 (2003) 605-620. doi:10.1163/156855203322448372.

[124] T. Han, H. Kalman, A. Levy, Theoretical and Experimental Study of Fatigue Strength of Particles, in: Third Isr. Conf. Conveying Handl. Part. Solids, 2000: p. 3.139-3.144.

[125] T. Han, Y. Petukhov, A. Levy, H. Kalman, Theoretical and experimental study of multi-impact breakage of particles, Adv. Powder Technol. 17 (2006) 135-157. doi:10.1163/156855206775992328.

[126] Y. Rozenblat, A. Levy, H. Kalman, I. Peyron, F. Ricard, A model for particle fatigue due to impact loads, Powder Technol. 239 (2013) 199-207. doi:10.1016/j.powtec.2013.01.059.

[127] H. Kalman, M. Hubert, E. Grant, Y. Petukhov, M. Haim, Fatigue behavior of impact comminution and attrition units, Powder Technol. 146 (2004) 1-9. doi:10.1016/j.powtec.2004.07.011.

[128] L.. Tavares, R.. King, Modeling of particle fracture by repeated impacts using continuum damage mechanics, Powder Technol. 123 (2002) 138-146. doi:10.1016/S00325910(01)00438-7.

[129] L.M. Tavares, Analysis of particle fracture by repeated stressing as damage accumulation, Powder Technol. 190 (2009) 327-339. doi:10.1016/j.powtec.2008.08.011.

[130] ASTM G76 - 13, Standard Test Method for Conducting Erosion Tests by Solid Particle Impingement Using Gas Jets, ASTM Int. (2013).

[131] DIN 50 332, Solid particle erosion test; basic rules, DIN. (1989).

[132] T. Deng, M.S. Bingley, M.S. a. Bradley, S.R. De Silva, A comparison of the gas-blast and centrifugal-accelerator erosion testers: The influence of particle dynamics, Wear. 265 (2008) 945-955. doi:10.1016/j.wear.2008.02.017.

[133] P.H. Shipway, I.M. Hutchings, Influence of nozzle roughness on conditions in a gas-blast erosion rig, Wear. 162-164 (1993) 148-158. doi:10.1016/0043-1648(93)90496-9.

[134] GOST 23.201-78, Products wear resistance assurance. Gas abrasive wear testing of materials and coatings with centrifugal accelerator, (1979).

[135] B.A. Kotzur, M.S.A. Bradley, R.J. Berry, R.J. Farnish, Breakage Characteristics of Granulated Food Products for Prediction of Attrition during Lean-Phase Pneumatic Conveying, Int. J. Food Eng. 12 (2016) 835-850.

[136] P. Chapelle, H. Abou-Chakra, N. Christakis, M. Patel, A. Abu-Nahar, U. Tüzün, M. Cross, Computational model for prediction of particle degradation during dilute-phase pneumatic 
conveying: the use of a laboratory-scale degradation tester for the determination of degradation propensity, Adv. Powder Technol. 15 (2004) 13-29.

doi:10.1163/15685520460740043.

[137] A.J. Burnett, M.S.A. Bradley, D.J. O'Flynn, T. Deng, M.S. Bingley, Anomalies in the results obtained from rotating disc accelerator erosion testers: A discussion of possible causes, Wear. 233-235 (1999) 275-283. doi:10.1016/S0043-1648(99)00227-6.

[138] A.J. Burnett, S.R. De Silva, A.R. Reed, Comparisons between "sand blast" and "centripetal effect accelerator" type erosion testers, Wear. 186-187 (1995) 168-178. doi:10.1016/00431648(95)07143-1.

[139] G.J. Brown, A fractal description of the progeny of double impact single particle breakage, Miner. Eng. 10 (1997) 229-235. doi:10.1016/S0892-6875(96)00148-3.

[140] D.. Weedon, F. Wilson, Modelling iron ore degradation using a twin pendulum breakage device, Int. J. Miner. Process. 59 (2000) 195-213. doi:10.1016/S0301-7516(99)00066-6.

[141] R.K. Sahoo, D.M. Weedon, D. Roach, Single-particle breakage tests of Gladstone Port Authority's coal by a twin pendulum apparatus, Adv. Powder Technol. 15 (2004) 263-280. doi:10.1163/156855204773644472.

[142] R.K. Sahoo, D.M. Weedon, D. Roach, Degradation model of Gladstone Port Authority's coal using a twin-pendulum apparatus, Adv. Powder Technol. 15 (2004) 459-475. doi:10.1163/1568552041270545.

[143] R. Sahoo, Review: An investigation of single particle breakage tests for coal handling system of the gladstone port, Powder Technol. 161 (2006) 158-167. doi:10.1016/j.powtec.2005.09.001.

[144] S.R. Krogh, Crushing characteristics, Powder Technol. 27 (1980) 171-181. doi:10.1016/00325910(80)85019-4.

[145] L.. Tavares, R.. King, Single-particle fracture under impact loading, Int. J. Miner. Process. 54 (1998) 1-28. doi:10.1016/S0301-7516(98)00005-2.

[146] L.M. Tavares, Energy absorbed in breakage of single particles in drop weight testing, Miner. Eng. 12 (1999) 43-50. doi:10.1016/S0892-6875(98)00118-6.

[147] L.M. Tavares, R.P. King, Measurement of the load-deformation response from impactbreakage of particles, Int. J. Miner. Process. 74 (2004) S267-S277. doi:10.1016/j.minpro.2004.07.017.

[148] G.J. Brown, N.J. Miles, T.F. Jones, A fractal description of the progeny of single impact single particle breakage, Miner. Eng. 9 (1996) 715-726. doi:10.1016/0892-6875(96)00063-5.

[149] B. Kotzur, M.S. Bradley, R.J. Berry, R.J. Farnish, Influence of Solids Loading Ratio on Particle Attrition within a New Centrifugal Accelerator Impact Tester, in: PARTEC Int. Congr. Part. Technol., 2016.

[150] S. Söderberg, S. Hogmark, U. Engman, H. Swahn, Erosion classification of materials using a centrifugal erosion tester, Tribol. Int. 14 (1981) 333-343. doi:10.1016/0301-679X(81)90101-8.

[151] E. Oveisi, A. Lau, S. Sokhansanj, C.J. Lim, X. Bi, S.H. Larsson, S. Melin, Breakage behavior of wood pellets due to free fall, Powder Technol. 235 (2013) 493-499. doi:10.1016/j.powtec.2012.10.022.

[152] Y. Petukhov, H. Kalman, A New Apparatus for Particle Impact Tests, Part. Part. Syst. Charact. 
20 (2003) 267-275. doi:10.1002/ppsc.200390032.

[153] The American Society of Agricultural and Biological Engineers, Densified Products for Bulk Handling - Definitions and Method, ASAE/ASABE S269.5. (2012) 1-8.

[154] M. Gentzler, J.N. Michaels, Impact attrition of brittle structured particles at low velocities: rigorous use of a laboratory vibrational impact tester, Chem. Eng. Sci. 59 (2004) 5949-5958. doi:10.1016/j.ces.2004.07.038.

[155] T. Han, A. Levy, H. Kalman, DEM simulation for attrition of salt during dilute-phase pneumatic conveying, Powder Technol. 129 (2003) 92-100. doi:10.1016/S0032-5910(02)00252-8.

[156] T.A. Bell, A. Boxman, J.B. Jacobs, Attrition of salt during pneumatic conveying, in: 5th World Congr. Chem. Eng., AIChE, San Diego, USA, 1996: pp. 238-243.

[157] D.G. Papadopoulos, C.S. Teo, M. Ghadiri, Attrition of common salt, in: 3rd World Congr. Part. Technol., Ichem, Brighton, 1998: p. Paper 156.

[158] K.J. Hanley, Experimental Quantification and Modelling of Attrition of Infant Formulae during Pneumatic Conveying, University College Cork, 2011.

[159] K.J. Hanley, K. Cronin, E.P. Byrne, Dispersion in particle velocity resulting from random motion through a spatially-varying fluid velocity field in a pipe, Powder Technol. 245 (2013) 255-264. doi:10.1016/j.powtec.2013.04.045.

[160] T. Brosh, H. Kalman, A. Levy, Fragments spawning and interaction models for DEM breakage simulation, Granul. Matter. 13 (2011) 765-776. doi:10.1007/s10035-011-0286-z.

[161] T. Brosh, H. Kalman, A. Levy, DEM simulation of particle attrition in dilute-phase pneumatic conveying, Granul. Matter. 13 (2011) 175-181. doi:10.1007/s10035-010-0201-z.

[162] T. Brosh, H. Kalman, A. Levy, Accelerating CFD-DEM simulation of processes with wide particle size distributions, Particuology. 12 (2014) 113-121. doi:10.1016/j.partic.2013.04.008.

[163] A. Levy, D.J. Mason, D. Levy-Hevroni, I. Borde, Drying of wet solid particles in a steady-state one-dimensional flow, Powder Technol. 95 (1998) 15-23. doi:10.1016/S0032-5910(97)033111.

[164] A.D. Salman, D.A. Gorham, M. Szabó, M.J. Hounslow, Spherical particle movement in dilute pneumatic conveying, Powder Technol. 153 (2005) 43-50. doi:10.1016/j.powtec.2005.01.023.

[165] C. Aked, D. Goder, H. Kalman, A. Zvieli, Attrition of very fine powders during pneumatic conveying, Powder Handl. Process. 9 (1997) 345-348. http://www.scopus.com/inward/record.url?eid=2-s2.0-0031246930\&partnerID=tZOtx3y1.

[166] H. Kalman, D. Goder, Design criteria for particle attrition, Adv. Powder Technol. 9 (1998) 153167. doi:10.1016/S0921-8831(08)60584-1.

[167] I. Bridle, S.R. Woodhead, A.R. Reed, The analysis of particle degradation in pneumatic conveyors utilizing a pilot-sized test facility, Proc. Inst. Mech. Eng. Part E J. Process Mech. 213 (1999) 85-91. https://www.scopus.com/inward/record.uri?eid=2-s2.00032831456\&partnerID=40\&md5=0c6eabce5d9a4287508943003c7908ef.

[168] L. Frye, W. Peukert, Attrition of Bulk Solids in Pneumatic Conveying: Mechanisms and Material Properties, Part. Sci. Technol. 20 (2002) 267-282. doi:10.1080/02726350216187.

[169] L. Frye, W. Peukert, Transfer of fracture mechanical concepts to bulk solids attrition in 
pneumatic conveying, Int. J. Miner. Process. 74 (2004) S279-S289.

doi:10.1016/j.minpro.2004.08.001.

[170] L. Frye, W. Peukert, Progress in the understanding of bulk solids attrition in dilute phase pneumatic conveying, Powder Technol. 143-144 (2004) 308-320.

doi:10.1016/j.powtec.2004.04.023.

[171] H. Abou-Chakra, U. Tüzün, I. Bridle, M. Leaper, M.S. a Bradley, a R. Reed, An investigation of particle degradation by impact within a centrifugal accelerator type degradation tester, Proc. Inst. Mech. Eng. Part E J. Process Mech. Eng. 217 (2003) 257-266.

doi:10.1243/095440803322328908.

[172] P. Chapelle, N. Christakis, H. Abou-Chakra, I. Bridle, M.S. a. Bradley, M. Patel, M. Cross, Computational model for prediction of particle degradation during dilute-phase pneumatic conveying: modeling of dilute-phase pneumatic conveying, Adv. Powder Technol. 15 (2004) 31-49. doi:10.1163/15685520460740052.

[173] W.J. Beekman, Measurement of the mechanical strength of granules and agglomerates, (2000). http://www.narcis.nl/publication/RecordID/oai\%3Atudelft.nl\%3Auuid\%3A242260634a23-4389-9e48-5419dd8e8e17 (accessed August 11, 2016).

[174] P. Gy, Sampling for Analytical Purposes, John Wiley and Sons, 1998.

[175] L. Petersen, P. Minkkinen, K.H. Esbensen, Representative sampling for reliable data analysis: Theory of Sampling, Chemom. Intell. Lab. Syst. 77 (2005) 261-277. doi:10.1016/j.chemolab.2004.09.013.

[176] H. Abou-Chakra, P. Chapelle, U. Tüzün, J. Baxter, M. Patel, M. Cross, I. Bridle, M.S.A. Bradley, Sampling Issues in Assessing Particle Degradation in Pneumatic Conveying Systems, Part. Part. Syst. Charact. 21 (2004) 39-46. doi:10.1002/ppsc.200400903.

[177] P. Gy, Sampling of discrete materials - a new introduction to the theory of sampling, Chemom. Intell. Lab. Syst. 74 (2004) 7-24. doi:10.1016/j.chemolab.2004.05.012.

[178] P. Gy, Sampling of discrete materials, Chemom. Intell. Lab. Syst. 74 (2004) 25-38. doi:10.1016/j.chemolab.2004.05.015.

[179] P. Gy, Sampling of discrete materials, Chemom. Intell. Lab. Syst. 74 (2004) 39-47. doi:10.1016/j.chemolab.2004.05.011.

[180] L. Petersen, C.K. Dahl, K.H. Esbensen, Representative mass reduction in sampling-a critical survey of techniques and hardware, Chemom. Intell. Lab. Syst. 74 (2004) 95-114. doi:10.1016/j.chemolab.2004.03.020.

[181] P. Kulkarni, Development of Bench-Scale Die Filling Tester for Free Flowing Particulates, University of Greenwich, 2012.

[182] A. Jillavenkatesa, S.J. Dapkunas, L.H. Lum, Particle Size Characterization, U.S. Department of Commerce, National Institute of Standards and Technology, Washington, D.C., 2001.

[183] M. Li, D. Wilkinson, K. Patchigolla, Comparison of Particle Size Distributions Measured Using Different Techniques, Part. Sci. Technol. 23 (2005) 265-284.

doi:10.1080/02726350590955912.

[184] P. Bowen, Particle Size Distribution Measurement from Millimeters to Nanometers and from Rods to Platelets, J. Dispers. Sci. Technol. 23 (2002) 631-662. doi:10.1081/DIS-120015368.

[185] Y. Petukhov, H. Kalman, Empirical breakage ratio of particles due to impact, Powder Technol. 
143-144 (2004) 160-169. doi:10.1016/j.powtec.2004.04.009.

[186] A.W. Jenike, Storage and Flow of Solids, 1964.

[187] B.A. Kotzur, M.S. a. Bradley, R.J. Berry, R.J. Farnish, Comparison of Relative Particle Breakage Levels for Large and Small Scale Centrifugal Attrition Testers, in: 8th Int. Conf. Conveying Handl. Part. Solids, Tel-Aviv, Israel, 2015. 